\title{
The Homoallyl-Cyclopropylcarbinyl Cation Manifold. Trimethylsilyl versus Aryl Stabilization
}

\author{
Xavier Creary,* Benjamin D. O'Donnell, and Marie Vervaeke \\ Department of Chemistry and Biochemistry \\ University of Notre Dame, Notre Dame, Indiana 46556
}

Supporting Information

Complete reference 10

B3LYP calculated structures, energies, and Cartesian coordinates

of $32,33,36,37$, and 38 .

S3-S7

${ }^{1} \mathrm{H}$ and ${ }^{13} \mathrm{C}$ NMR spectra of compounds 13, 14, 15, $9\left(\mathrm{Ar}=\mathrm{C}_{6} \mathrm{H}_{5}\right), 9(\mathrm{Ar}=$ $\left.p-\mathrm{CH}_{3} \mathrm{C}_{6} \mathrm{H}_{4}\right), 9\left(\mathrm{Ar}=p-\mathrm{ClC}_{6} \mathrm{H}_{4}\right), 17$ and 18, $19\left(\mathrm{Ar}=\mathrm{C}_{6} \mathrm{H}_{5}\right), \mathbf{2 0}, 21$, and 22.

${ }^{1} \mathrm{H}$ NMR spectra of products from reaction of 9 in $\mathrm{CD}_{3} \mathrm{CO}_{2} \mathrm{D}$.

S30-S34

${ }^{1} \mathrm{H}$ NMR spectra of products from reaction of 17 and 18 in $\mathrm{CD}_{3} \mathrm{CO}_{2} \mathrm{D}$.

First order kinetic plot for reaction of $\mathbf{1 0}\left(\mathrm{Ar}=\mathrm{C}_{6} \mathrm{H}_{5}\right)$ in $\mathrm{CD}_{3} \mathrm{CO}_{2} \mathrm{D}$ along with corresponding ${ }^{1} \mathrm{H}$ NMR spectra. 
10. Frisch, M, J.; Trucks, G. W.; Schlegel, H. B.; Scuseria, G. E.; Robb, M. A.; Cheeseman, J. R.; Montgomery, Jr., J. A.; Vreven, T.; Kudin, K. N.; Burant, J. C.; Millam, J. M.; Iyengar, S. S.; Tomasi, J.; Barone, V.; Mennucci, B.; Cossi, M.; Scalmani, G.; Rega, N.; Petersson, G. A.; Nakatsuji, H.; Hada, M.; Ehara, M.; Toyota, K.; Fukuda, R.; Hasegawa, J.; Ishida, M.; Nakajima, T.; Honda, Y.; Kitao, O.; Nakai, H.; Klene, M.; Li, X.; Knox, J. E.; Hratchian, H. P.; Cross, J. B.; C. Adamo, C.; Jaramillo, J.; Gomperts, R.; Stratmann, R. E.; Yazyev, O.; A. J. Austin, A. J.; R. Cammi, R.; Pomelli, C.; Ochterski, J. W.; Ayala, P. Y.; Morokuma, K.; Voth, G. A.; Salvador, P.; Dannenberg, J. J.; Zakrzewski, V. G.; Dapprich, S.; Daniels, A. D.; Strain, M. C.; Farkas, O.; Malick, D. K.; Rabuck, A. D.; Raghavachari, K.; Foresman, J. B.; Ortiz, J. V.; Cui, Q.; Baboul, A. G.; Clifford, S.; Cioslowski, J.; Stefanov, B. B.; Liu, G.; Liashenko, A.; Piskorz, P.; Komaromi, I.; Martin, R. L.; Fox, D. J.; Keith, T.; Al-Laham, M. A.; Peng, C. Y.; Nanayakkara, A.; Challacombe, M.; Gill, P. M. W.; Johnson, B.; Chen, W.; Wong, M. W.; Gonzalez, C.; Pople, J. A. Gaussian 03, Revision C.01, Gaussian, Inc., Wallingford CT, 2004. 


\section{B3LYP/6-31G* Optimized Structure and Energy of 32}

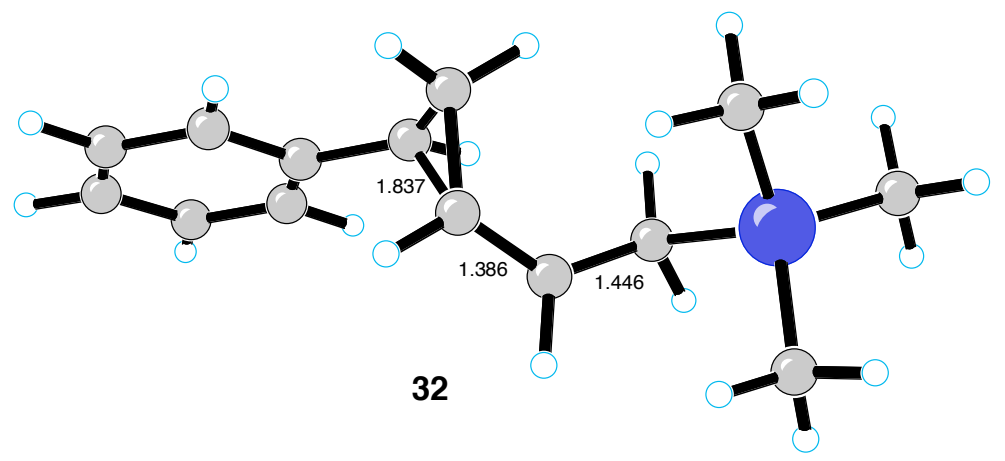

$E(R B+H F-L Y P)=-835.399103$

Zero-point correction $=0.310505$ au No Imaginary Frequencies

\begin{tabular}{|c|c|c|c|c|c|}
\hline \multirow{2}{*}{$\begin{array}{l}\text { Center } \\
\text { Number }\end{array}$} & \multirow{2}{*}{$\begin{array}{l}\text { Atomic } \\
\text { Number }\end{array}$} & \multirow{2}{*}{$\begin{array}{c}\text { Atomic } \\
\text { Type }\end{array}$} & \multicolumn{3}{|c|}{ Coordinates (Angstroms) } \\
\hline & & & $\mathrm{X}$ & $\mathrm{Y}$ & $\mathrm{Z}$ \\
\hline 1 & 6 & 0 & -1.221028 & 0.773736 & -0.583286 \\
\hline 2 & 6 & 0 & -0.718833 & -0.201672 & 0.472202 \\
\hline 3 & 6 & 0 & 0.484624 & -0.886870 & 0.411090 \\
\hline 4 & 1 & 0 & -1.396258 & -0.414508 & 1.291396 \\
\hline 5 & 6 & 0 & 1.500934 & -0.837652 & -0.616257 \\
\hline 6 & 1 & 0 & 0.663442 & -1.587989 & 1.226703 \\
\hline 7 & 1 & 0 & 2.508299 & -0.947014 & -0.198508 \\
\hline 8 & 1 & 0 & 1.458652 & 0.037796 & -1.271436 \\
\hline 9 & 14 & 0 & 1.377913 & -2.424995 & -1.824457 \\
\hline 10 & 6 & 0 & -0.260812 & -2.329755 & -2.747103 \\
\hline 11 & 6 & 0 & 2.853758 & -2.227139 & -2.974265 \\
\hline 12 & 6 & 0 & 1.503402 & -3.969809 & -0.757382 \\
\hline 13 & 1 & 0 & -0.336001 & -1.425684 & -3.362752 \\
\hline 14 & 1 & 0 & -1.122723 & -2.359841 & -2.070888 \\
\hline 15 & 1 & 0 & -0.352660 & -3.186856 & -3.425377 \\
\hline 16 & 1 & 0 & 3.802946 & -2.239993 & -2.426939 \\
\hline 17 & 1 & 0 & 2.805801 & -1.296251 & -3.550435 \\
\hline 18 & 1 & 0 & 2.877731 & -3.056025 & -3.692363 \\
\hline 19 & 1 & 0 & 2.406366 & -3.968408 & -0.135687 \\
\hline 20 & 1 & 0 & 1.554796 & -4.858733 & -1.397676 \\
\hline 21 & 1 & 0 & 0.636072 & -4.097119 & -0.099554 \\
\hline 22 & 1 & 0 & -0.777636 & 0.660428 & -1.569307 \\
\hline 23 & 6 & 0 & -0.501220 & 1.619747 & 0.367182 \\
\hline 24 & 1 & 0 & -2.304292 & 0.856623 & -0.619148 \\
\hline 25 & 1 & 0 & 0.550136 & 1.790804 & 0.154693 \\
\hline 26 & 6 & 0 & -1.062207 & 2.393493 & 1.456070 \\
\hline 27 & 6 & 0 & -2.041683 & 3.986181 & 3.542414 \\
\hline 28 & 6 & 0 & -2.449013 & 2.452429 & 1.719700 \\
\hline 29 & 6 & 0 & -0.179286 & 3.146941 & 2.261395 \\
\hline 30 & 6 & 0 & -0.666661 & 3.940322 & 3.292451 \\
\hline 31 & 6 & 0 & -2.929890 & 3.241904 & 2.755410 \\
\hline 32 & 1 & 0 & -3.151179 & 1.893057 & 1.108863 \\
\hline 33 & 1 & 0 & 0.889044 & 3.108127 & 2.064659 \\
\hline 34 & 1 & 0 & 0.018789 & 4.521517 & 3.900956 \\
\hline 35 & 1 & 0 & -3.996853 & 3.288109 & 2.949239 \\
\hline 36 & 1 & 0 & -2.424452 & 4.605101 & 4.348397 \\
\hline
\end{tabular}




\section{B3LYP/6-31G* Optimized Structure and Energy of 33}

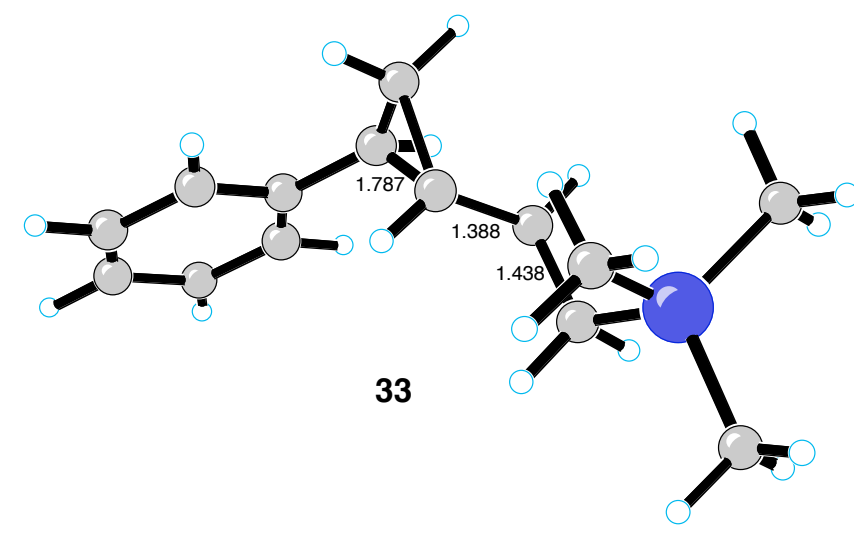

$E(R B+H F-L Y P)=-835.401366$

Zero-point correction $=0.310441 \mathrm{au}$ No Imaginary Frequencies

\begin{tabular}{|c|c|c|c|c|c|}
\hline \multirow{2}{*}{$\begin{array}{l}\text { Center } \\
\text { Number }\end{array}$} & \multirow{2}{*}{$\begin{array}{l}\text { Atomic } \\
\text { Number }\end{array}$} & \multirow{2}{*}{$\begin{array}{c}\text { Atomic } \\
\text { Type }\end{array}$} & \multicolumn{3}{|c|}{ Coordinates (Angstroms) } \\
\hline & & & $\mathrm{X}$ & $\mathrm{Y}$ & $\mathrm{Z}$ \\
\hline 1 & 1 & 0 & -1.498114 & 2.215247 & -0.280826 \\
\hline $\begin{array}{l}1 \\
2\end{array}$ & $\begin{array}{l}1 \\
6\end{array}$ & 0 & -1.733207 & 1.411729 & 0.410656 \\
\hline 3 & 6 & 0 & -2.282955 & 0.193595 & -0.182584 \\
\hline 4 & 6 & 0 & -0.784045 & -0.035528 & -0.032146 \\
\hline 5 & 6 & 0 & -1.698055 & 1.768395 & 1.823732 \\
\hline 6 & 1 & 0 & -2.646782 & 0.266780 & -1.204637 \\
\hline 7 & 1 & 0 & -2.889955 & -0.455218 & 0.443193 \\
\hline 8 & 6 & 0 & 0.138559 & 0.266612 & -1.024550 \\
\hline 9 & 1 & 0 & -0.452671 & -0.550908 & 0.863756 \\
\hline 10 & 6 & 0 & -1.646342 & 2.586608 & 4.503379 \\
\hline 11 & 6 & 0 & -1.181215 & 3.031555 & 2.178347 \\
\hline 12 & 6 & 0 & -2.186037 & 0.918895 & 2.838698 \\
\hline 13 & 6 & 0 & -2.158113 & 1.327820 & 4.166113 \\
\hline 14 & 6 & 0 & -1.160989 & 3.438794 & 3.507693 \\
\hline 15 & 1 & 0 & -0.804210 & 3.693875 & 1.403134 \\
\hline 16 & 1 & 0 & -2.600373 & -0.054564 & 2.592565 \\
\hline 17 & 1 & 0 & -2.542508 & 0.671966 & 4.940972 \\
\hline 18 & 1 & 0 & -0.769024 & 4.416732 & 3.768712 \\
\hline 19 & 1 & 0 & -1.630761 & 2.902776 & 5.542056 \\
\hline 20 & 6 & 0 & 1.526857 & -0.108748 & -1.031351 \\
\hline 21 & 1 & 0 & -0.231576 & 0.806839 & -1.898153 \\
\hline 22 & 1 & 0 & 1.922460 & -0.358962 & -0.041967 \\
\hline 23 & 1 & 0 & 2.161221 & 0.627884 & -1.536037 \\
\hline 24 & 14 & 0 & 1.823269 & -1.758573 & -2.132009 \\
\hline 25 & 6 & 0 & 1.158444 & -1.429206 & -3.861970 \\
\hline 26 & 6 & 0 & 3.691444 & -1.970379 & -2.110714 \\
\hline 27 & 6 & 0 & 0.924763 & -3.173187 & -1.276859 \\
\hline 28 & 1 & 0 & 1.099645 & -4.107192 & -1.824630 \\
\hline 29 & 1 & 0 & -0.159565 & -3.018030 & -1.240624 \\
\hline 30 & 1 & 0 & 1.283263 & -3.327777 & -0.252617 \\
\hline 31 & 1 & 0 & 1.391544 & -2.278506 & -4.515674 \\
\hline 32 & 1 & 0 & 1.612298 & -0.540517 & -4.315787 \\
\hline 33 & 1 & 0 & 0.069758 & -1.302224 & -3.876845 \\
\hline 34 & 1 & 0 & 3.969950 & -2.865663 & -2.680124 \\
\hline 35 & 1 & 0 & 4.079823 & -2.094061 & -1.093686 \\
\hline 36 & 1 & 0 & 4.203186 & -1.116857 & -2.569219 \\
\hline
\end{tabular}




\section{B3LYP/6-31G* Optimized Structure and Energy of $\mathbf{3 6}$}

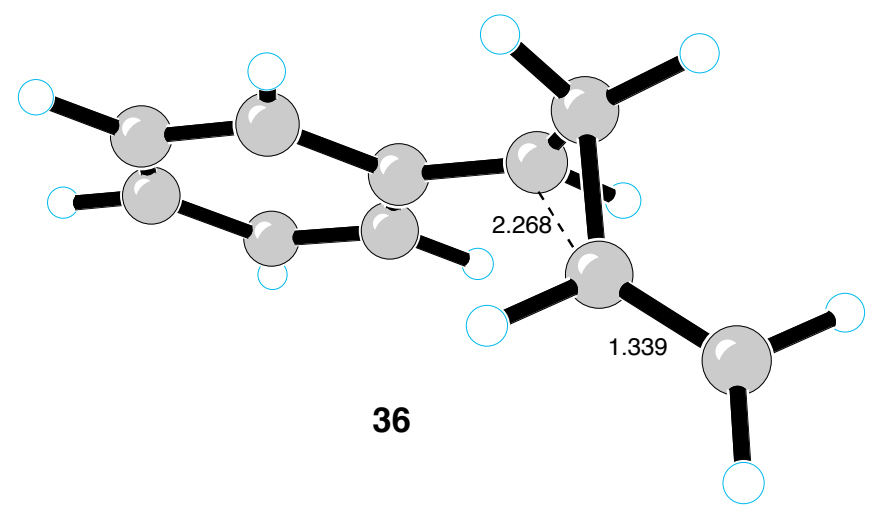

$E(R B+H F-L Y P)=-387.389818$

Zero-point correction $=0.179775$ au

No Imaginary Frequencies

\begin{tabular}{|c|c|c|c|c|c|}
\hline \multirow{2}{*}{$\begin{array}{l}\text { Center } \\
\text { Number }\end{array}$} & \multirow{2}{*}{$\begin{array}{l}\text { Atomic } \\
\text { Number }\end{array}$} & \multirow{2}{*}{$\begin{array}{c}\text { Atomic } \\
\text { Type }\end{array}$} & \multicolumn{3}{|c|}{ Coordinates (Angstroms) } \\
\hline & & & $\mathrm{X}$ & $\mathrm{Y}$ & Z \\
\hline 1 & 1 & 0 & -1.287939 & -0.406363 & 1.615995 \\
\hline 2 & 6 & 0 & -0.314592 & -0.487699 & 1.133299 \\
\hline 3 & 6 & 0 & 0.826753 & -0.635659 & 2.055715 \\
\hline 4 & 6 & 0 & -0.295423 & -0.337884 & -0.260010 \\
\hline 5 & 6 & 0 & -0.386707 & -0.096887 & -3.043025 \\
\hline 6 & 6 & 0 & -1.534209 & -0.086347 & -0.929833 \\
\hline 7 & 6 & 0 & 0.899490 & -0.462526 & -1.035598 \\
\hline 8 & 6 & 0 & 0.846162 & -0.341078 & -2.409625 \\
\hline 9 & 6 & 0 & -1.574931 & 0.029119 & -2.306501 \\
\hline 10 & 1 & 0 & -2.443001 & 0.009587 & -0.342287 \\
\hline 11 & 1 & 0 & 1.847435 & -0.660142 & -0.546867 \\
\hline 12 & 1 & 0 & 1.748863 & -0.438135 & -3.003584 \\
\hline 13 & 1 & 0 & -2.514335 & 0.216249 & -2.815768 \\
\hline 14 & 1 & 0 & -0.418639 & -0.006373 & -4.125229 \\
\hline 15 & 6 & 0 & 0.973397 & 0.841169 & 2.444797 \\
\hline 16 & 1 & 0 & 1.738188 & -1.023517 & 1.598361 \\
\hline 17 & 1 & 0 & 0.562109 & -1.241768 & 2.925437 \\
\hline 18 & 6 & 0 & 0.379609 & 1.387588 & 3.513763 \\
\hline 19 & 1 & 0 & 1.560819 & 1.448218 & 1.760363 \\
\hline 20 & 1 & 0 & -0.241033 & 0.809127 & 4.194283 \\
\hline 21 & 1 & 0 & 0.530237 & 2.434332 & 3.761398 \\
\hline
\end{tabular}




\section{B3LYP/6-31G* Optimized Structure and Energy of 37}

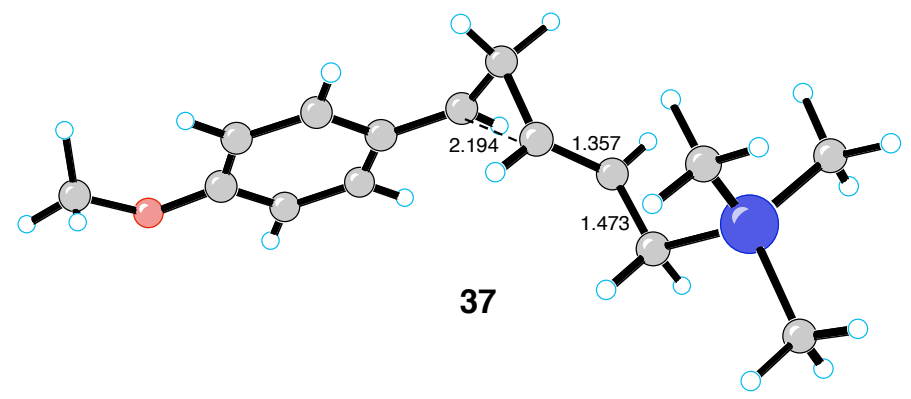

$E(R B+H F-L Y P)=-949.935052254$

Zero-point correction $=0.343175$ au No Imaginary Frequencies

\begin{tabular}{|c|c|c|c|c|c|}
\hline \multirow{2}{*}{$\begin{array}{l}\text { Center } \\
\text { Number }\end{array}$} & \multirow{2}{*}{$\begin{array}{l}\text { Atomic } \\
\text { Number }\end{array}$} & \multirow{2}{*}{$\begin{array}{l}\text { Atomic } \\
\text { Type }\end{array}$} & \multicolumn{3}{|c|}{ Coordinates (Angstroms) } \\
\hline & & & $\mathrm{X}$ & $\mathrm{Y}$ & $\mathrm{Z}$ \\
\hline 1 & 1 & 0 & -1.472286 & 1.991647 & -0.734427 \\
\hline 2 & 6 & 0 & -1.660671 & 1.231894 & 0.021725 \\
\hline 3 & 6 & 0 & -1.892927 & -0.128934 & -0.506927 \\
\hline 4 & 6 & 0 & -0.410562 & -0.430210 & -0.676518 \\
\hline 5 & 6 & 0 & -1.566764 & 1.642906 & 1.356920 \\
\hline 6 & 1 & 0 & -2.424952 & -0.117870 & -1.460462 \\
\hline 7 & 1 & 0 & -2.387125 & -0.817014 & 0.180521 \\
\hline 8 & 6 & 0 & 0.285137 & -0.169161 & -1.811829 \\
\hline 9 & 1 & 0 & 0.102797 & -0.803904 & 0.206660 \\
\hline 10 & 6 & 0 & -1.445742 & 2.589989 & 4.009743 \\
\hline 11 & 6 & 0 & -1.207676 & 3.000149 & 1.643922 \\
\hline 12 & 6 & 0 & -1.854896 & 0.783000 & 2.460389 \\
\hline 13 & 6 & 0 & -1.797598 & 1.237856 & 3.757021 \\
\hline 14 & 6 & 0 & -1.151995 & 3.464808 & 2.931168 \\
\hline 15 & 1 & 0 & -0.982204 & 3.670019 & 0.818863 \\
\hline 16 & 1 & 0 & -2.132148 & -0.250448 & 2.279993 \\
\hline 17 & 1 & 0 & -2.026518 & 0.566815 & 4.575777 \\
\hline 18 & 1 & 0 & -0.887947 & 4.491291 & 3.160698 \\
\hline 19 & 8 & 0 & -1.366442 & 3.129938 & 5.216725 \\
\hline 20 & 6 & 0 & 1.704720 & -0.483565 & -2.050284 \\
\hline 21 & 1 & 0 & -0.262781 & 0.258586 & -2.654399 \\
\hline 22 & 1 & 0 & 2.250385 & -0.638833 & -1.112027 \\
\hline 23 & 1 & 0 & 2.189897 & 0.331318 & -2.604388 \\
\hline 24 & 14 & 0 & 1.984611 & -2.073583 & -3.151170 \\
\hline 25 & 6 & 0 & 1.083136 & -1.831775 & -4.792484 \\
\hline 26 & 6 & 0 & 3.848035 & -2.203389 & -3.408915 \\
\hline 27 & 6 & 0 & 1.314474 & -3.570426 & -2.217132 \\
\hline 28 & 1 & 0 & 1.464041 & -4.484875 & -2.803375 \\
\hline 29 & 1 & 0 & 0.240333 & -3.482103 & -2.017314 \\
\hline 30 & 1 & 0 & 1.825927 & -3.712168 & -1.257564 \\
\hline 31 & 1 & 0 & 1.286343 & -2.672145 & -5.466913 \\
\hline 32 & 1 & 0 & 1.409830 & -0.919015 & -5.304921 \\
\hline 33 & 1 & 0 & -0.004428 & -1.776501 & -4.664677 \\
\hline 34 & 1 & 0 & 4.091412 & -3.088595 & -4.008721 \\
\hline 35 & 1 & 0 & 4.385876 & -2.294014 & -2.458024 \\
\hline 36 & 1 & 0 & 4.246123 & -1.329541 & -3.937700 \\
\hline 37 & 6 & 0 & -1.646543 & 2.349829 & 6.395014 \\
\hline 38 & 1 & 0 & -2.678029 & 1.985560 & 6.377283 \\
\hline 39 & 1 & 0 & -1.509410 & 3.036683 & 7.228602 \\
\hline 40 & 1 & 0 & -0.943441 & 1.515781 & 6.479146 \\
\hline
\end{tabular}


B3LYP/6-31G* Optimized Structure and Energy of $\mathbf{3 8}$

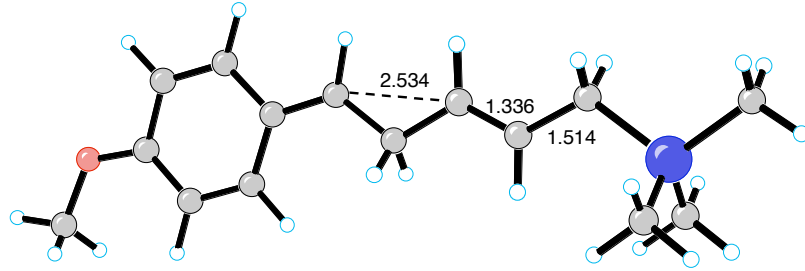

38

$E(R B+H F-L Y P)=-949.917203$

Zero-point correction $=0.340748$ au

3 Imaginary Frequencies

\begin{tabular}{|c|c|c|c|c|c|}
\hline \multirow{2}{*}{$\begin{array}{l}\text { Center } \\
\text { Number }\end{array}$} & \multirow{2}{*}{$\begin{array}{l}\text { Atomic } \\
\text { Number }\end{array}$} & \multirow{2}{*}{$\begin{array}{l}\text { Atomic } \\
\text { Type }\end{array}$} & \multicolumn{3}{|c|}{ Coordinates (Angstroms) } \\
\hline & & & $\mathrm{X}$ & $\mathrm{Y}$ & $\mathrm{Z}$ \\
\hline 1 & 1 & 0 & -2698940 & $-0,000000$ & 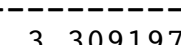 \\
\hline $\begin{array}{l}1 \\
2\end{array}$ & $\frac{1}{6}$ & 0 & -1.656025 & -0.000000 & 3.613169 \\
\hline 3 & 6 & 0 & 1.070395 & 0.000000 & 4.348263 \\
\hline 4 & 6 & 0 & -0.636806 & -0.000000 & 2.594405 \\
\hline 5 & 6 & 0 & -1.333000 & -0.000000 & 4.940074 \\
\hline 6 & 6 & 0 & 0.035908 & -0.000000 & 5.331629 \\
\hline 7 & 6 & 0 & 0.736426 & 0.000000 & 3.020894 \\
\hline 8 & 6 & 0 & -1.012534 & -0.000000 & 1.263329 \\
\hline 9 & 1 & 0 & -2.087739 & -0.000000 & 5.718725 \\
\hline 10 & 8 & 0 & 0.249760 & 0.000000 & 6.629664 \\
\hline 11 & 1 & 0 & 1.526192 & 0.000000 & 2.276614 \\
\hline 12 & 1 & 0 & 2.111092 & 0.000000 & 4.648342 \\
\hline 13 & 6 & 0 & -0.135063 & -0.000000 & 0.065165 \\
\hline 14 & 1 & 0 & -2.086742 & -0.000000 & 1.071701 \\
\hline 15 & 1 & 0 & 0.541280 & 0.869188 & 0.140292 \\
\hline 16 & 6 & 0 & -0.857060 & -0.000000 & -1.266207 \\
\hline 17 & 1 & 0 & 0.541280 & -0.869188 & 0.140292 \\
\hline 18 & 6 & 0 & -0.228954 & -0.000000 & -2.445731 \\
\hline 19 & 1 & 0 & -1.947356 & -0.000000 & -1.232921 \\
\hline 20 & 1 & 0 & 0.864341 & 0.000000 & -2.433981 \\
\hline 21 & 6 & 0 & -0.913993 & -0.000000 & -3.795506 \\
\hline 22 & 6 & 0 & 1.588527 & 0.000000 & 7.177038 \\
\hline 23 & 1 & 0 & 2.124000 & 0.901252 & 6.866730 \\
\hline 24 & 1 & 0 & 2.124000 & -0.901252 & 6.866730 \\
\hline 25 & 1 & 0 & 1.448920 & 0.000000 & 8.256408 \\
\hline 26 & 14 & 0 & 0.240773 & 0.000000 & -5.325475 \\
\hline 27 & 1 & 0 & -1.579073 & 0.873245 & -3.867169 \\
\hline 28 & 1 & 0 & -1.579073 & -0.873245 & -3.867169 \\
\hline 29 & 6 & 0 & -0.844172 & -0.000000 & -6.871279 \\
\hline 30 & 6 & 0 & 1.324517 & 1.551487 & -5.274328 \\
\hline 31 & 6 & 0 & 1.324517 & -1.551487 & -5.274328 \\
\hline 32 & 1 & 0 & -0.231897 & -0.000000 & -7.780858 \\
\hline 33 & 1 & 0 & -1.490288 & 0.885104 & -6.911705 \\
\hline 34 & 1 & 0 & -1.490288 & -0.885104 & -6.911705 \\
\hline 35 & 1 & 0 & 1.999851 & -1.584918 & -6.137566 \\
\hline 36 & 1 & 0 & 0.718654 & -2.465330 & -5.297645 \\
\hline 37 & 1 & 0 & 1.949427 & -1.590966 & -4.373455 \\
\hline 38 & 1 & 0 & 1.999851 & 1.584918 & -6.137566 \\
\hline 39 & 1 & 0 & 1.949427 & 1.590966 & -4.373455 \\
\hline 40 & 1 & 0 & 0.718654 & 2.465330 & -5.297645 \\
\hline
\end{tabular}




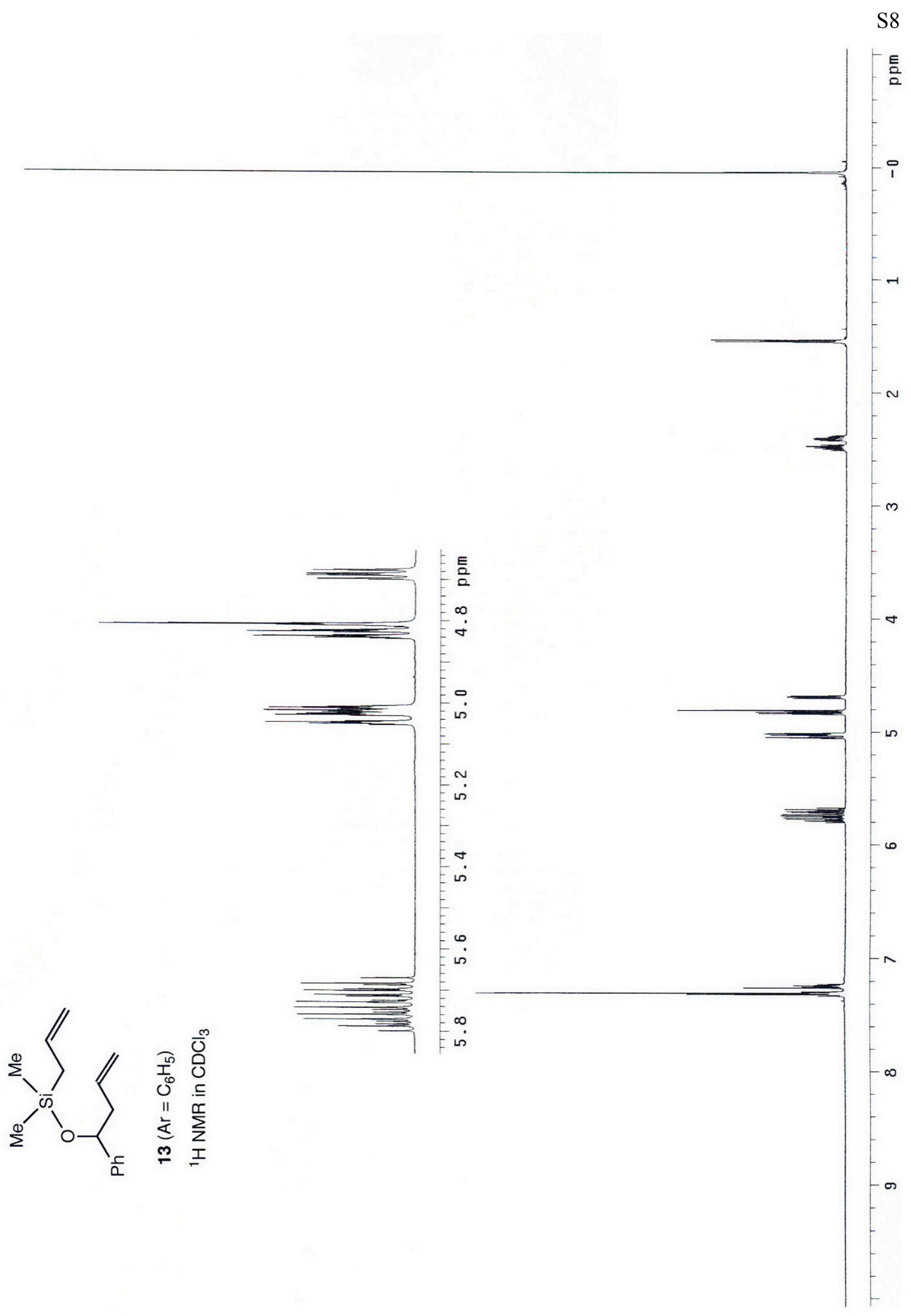




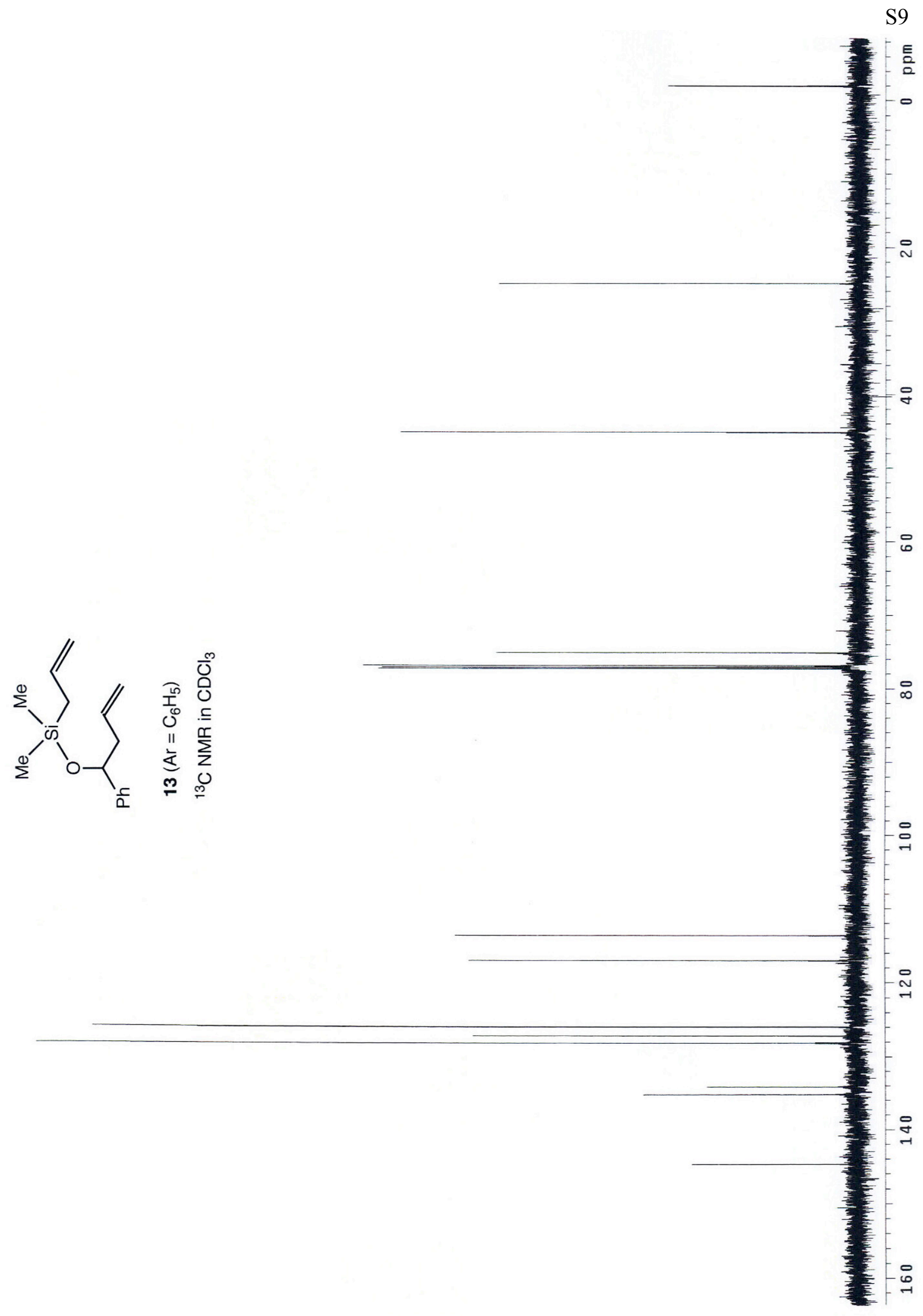




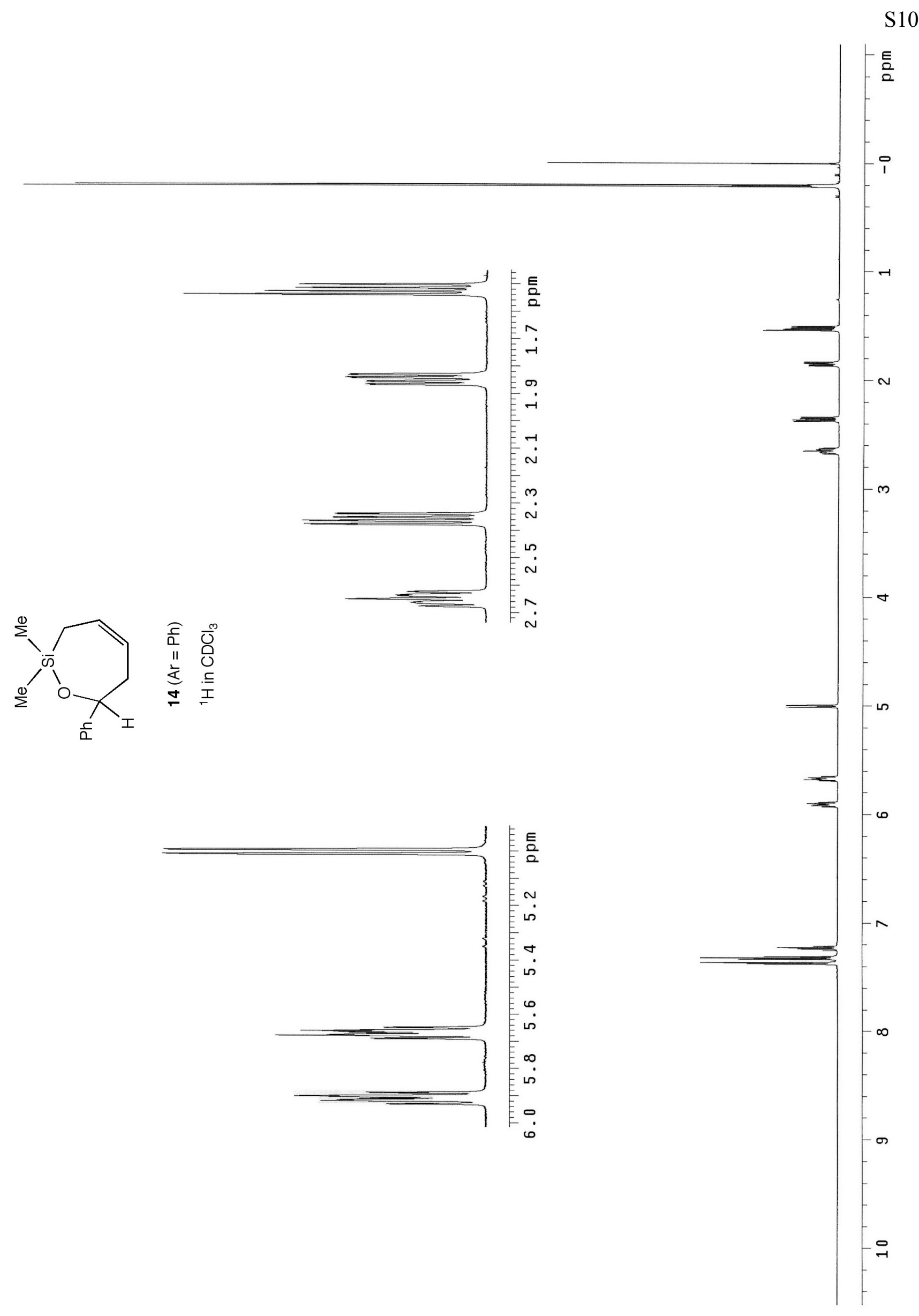


S11

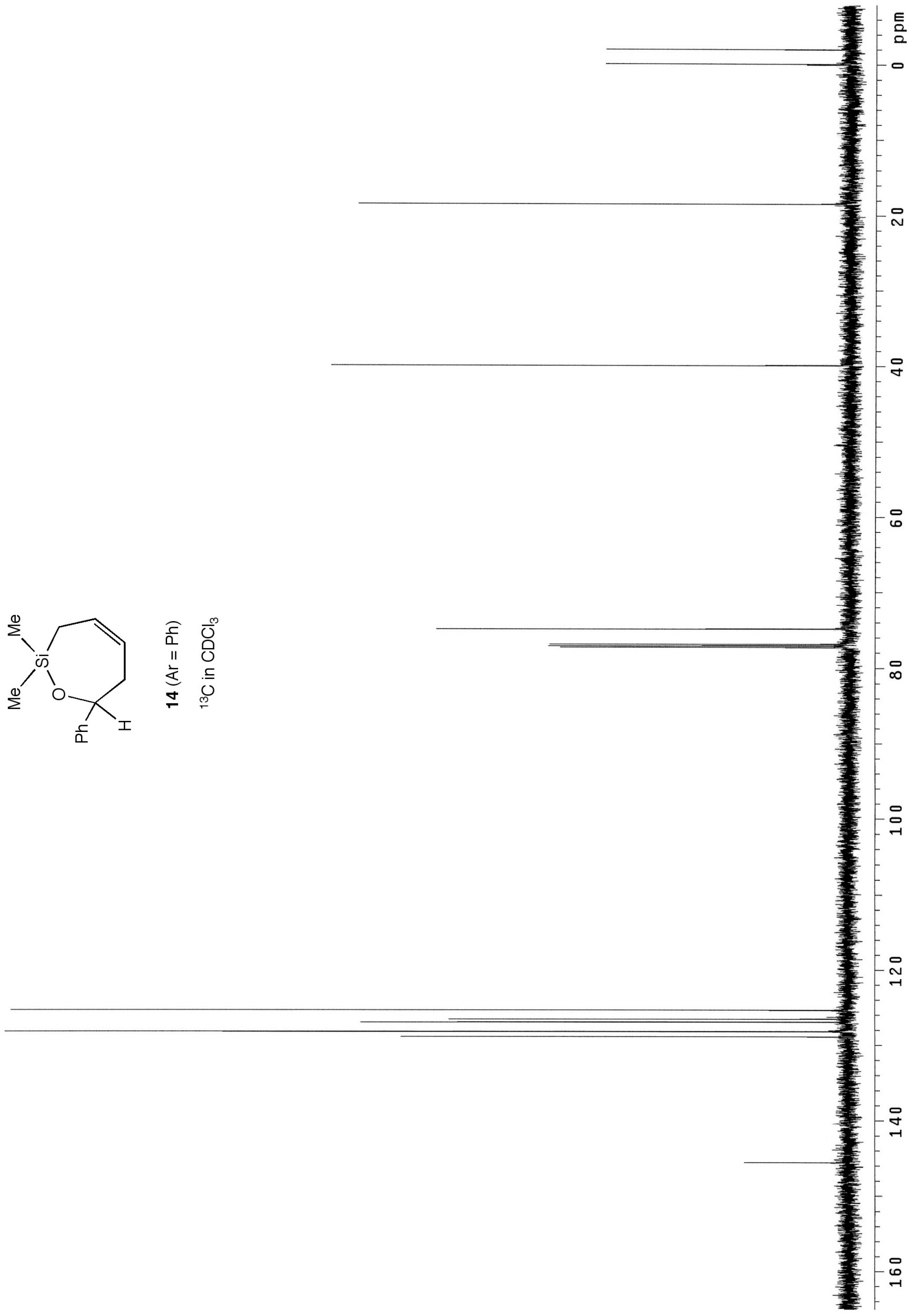




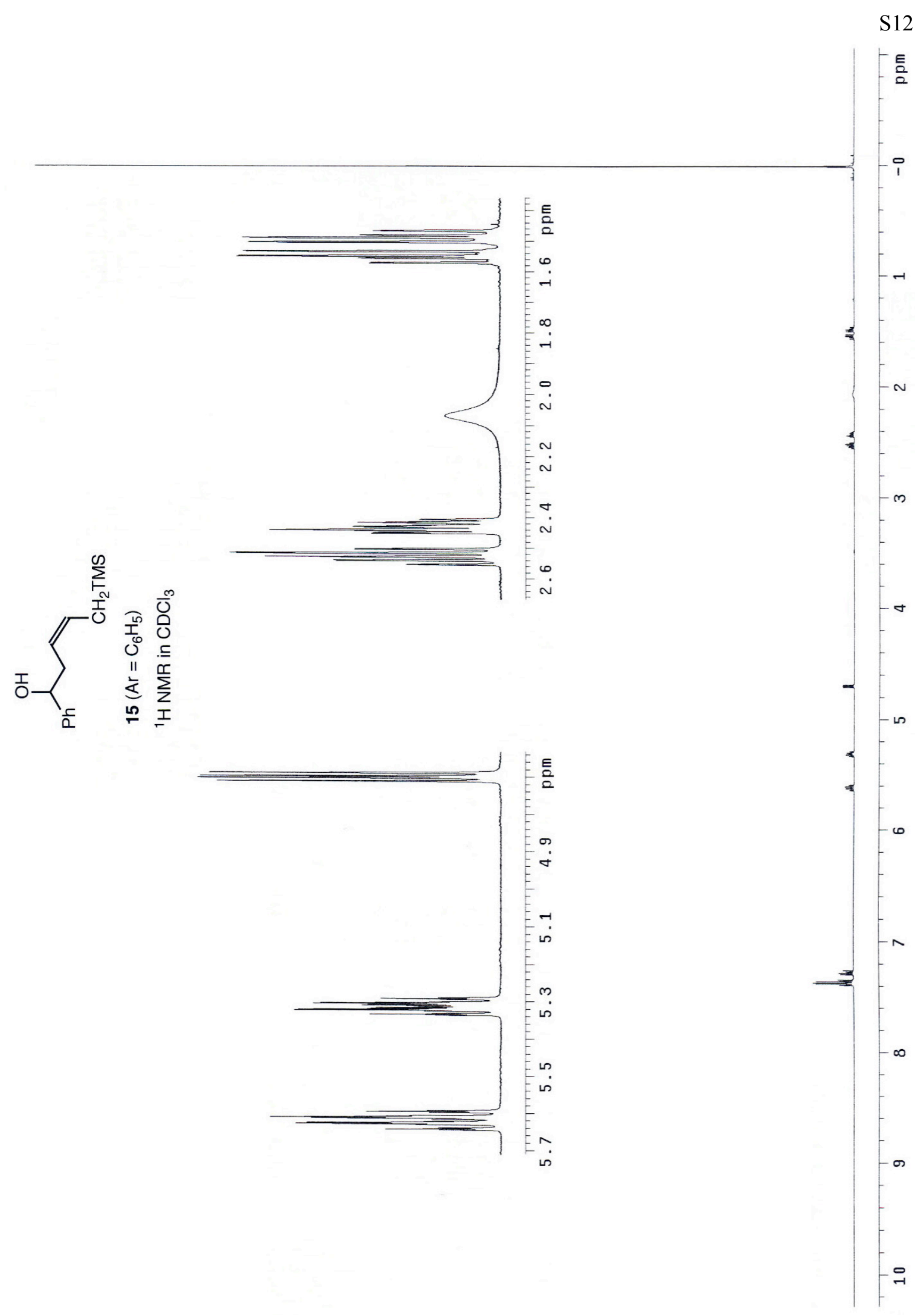



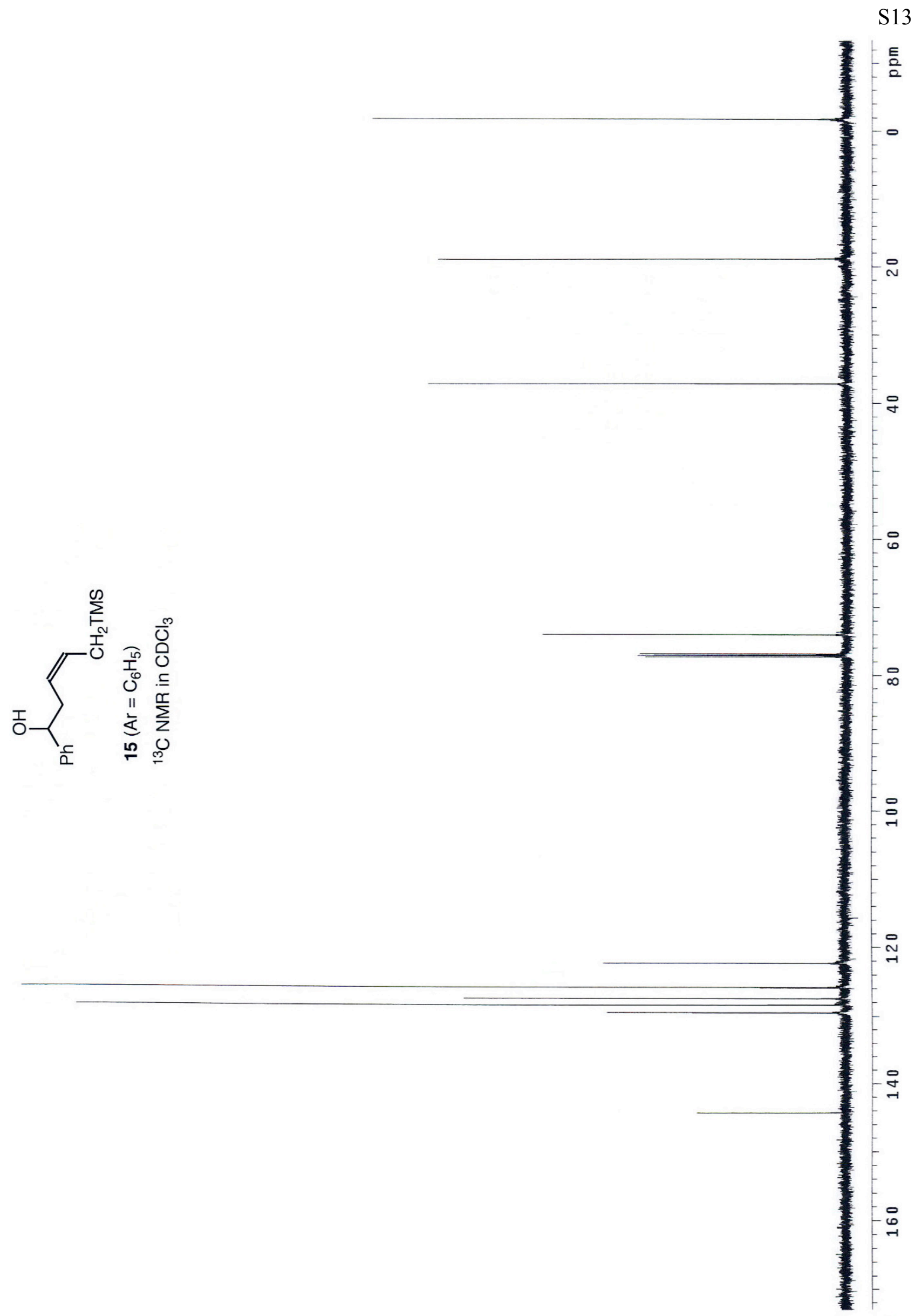


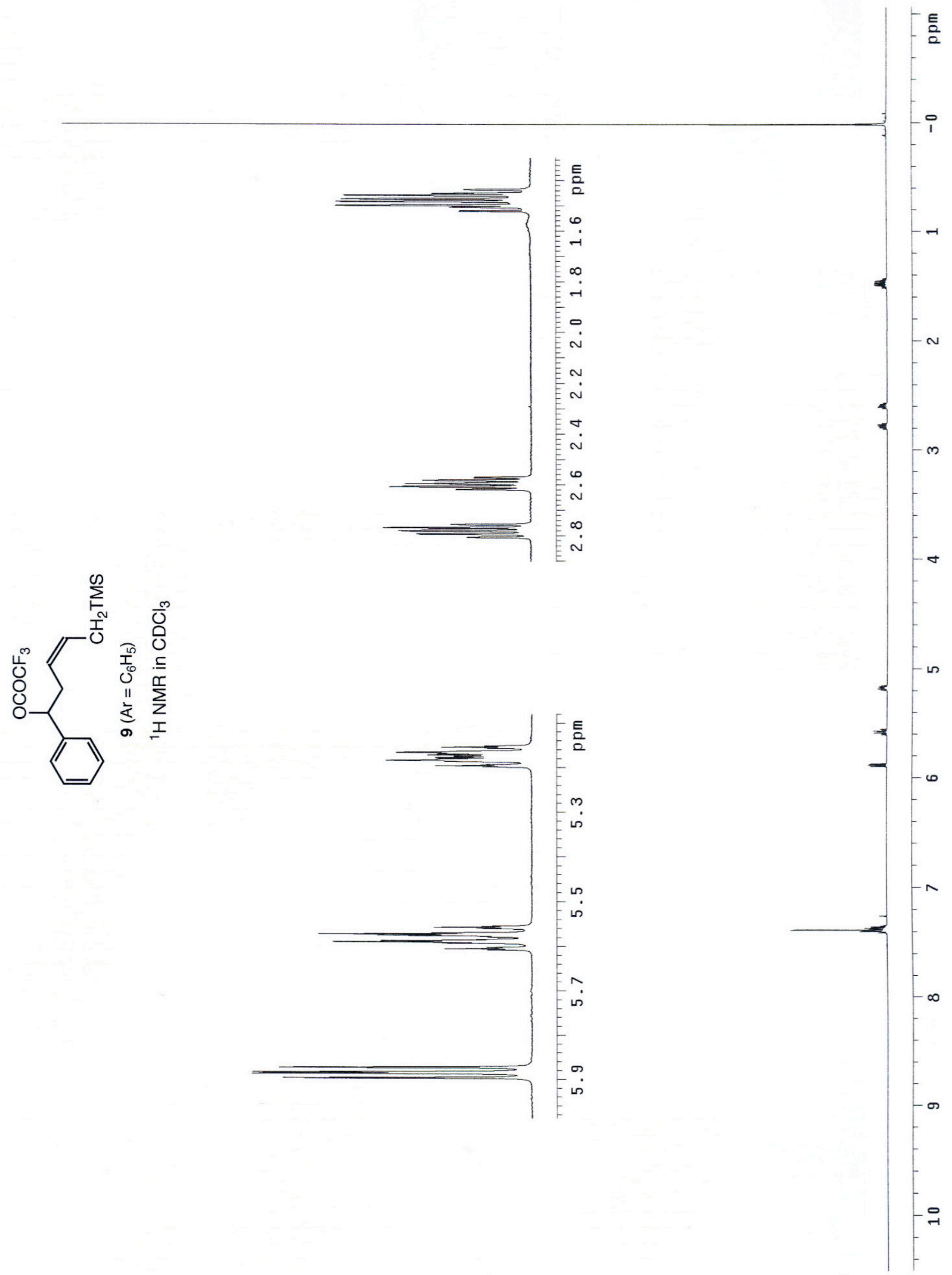




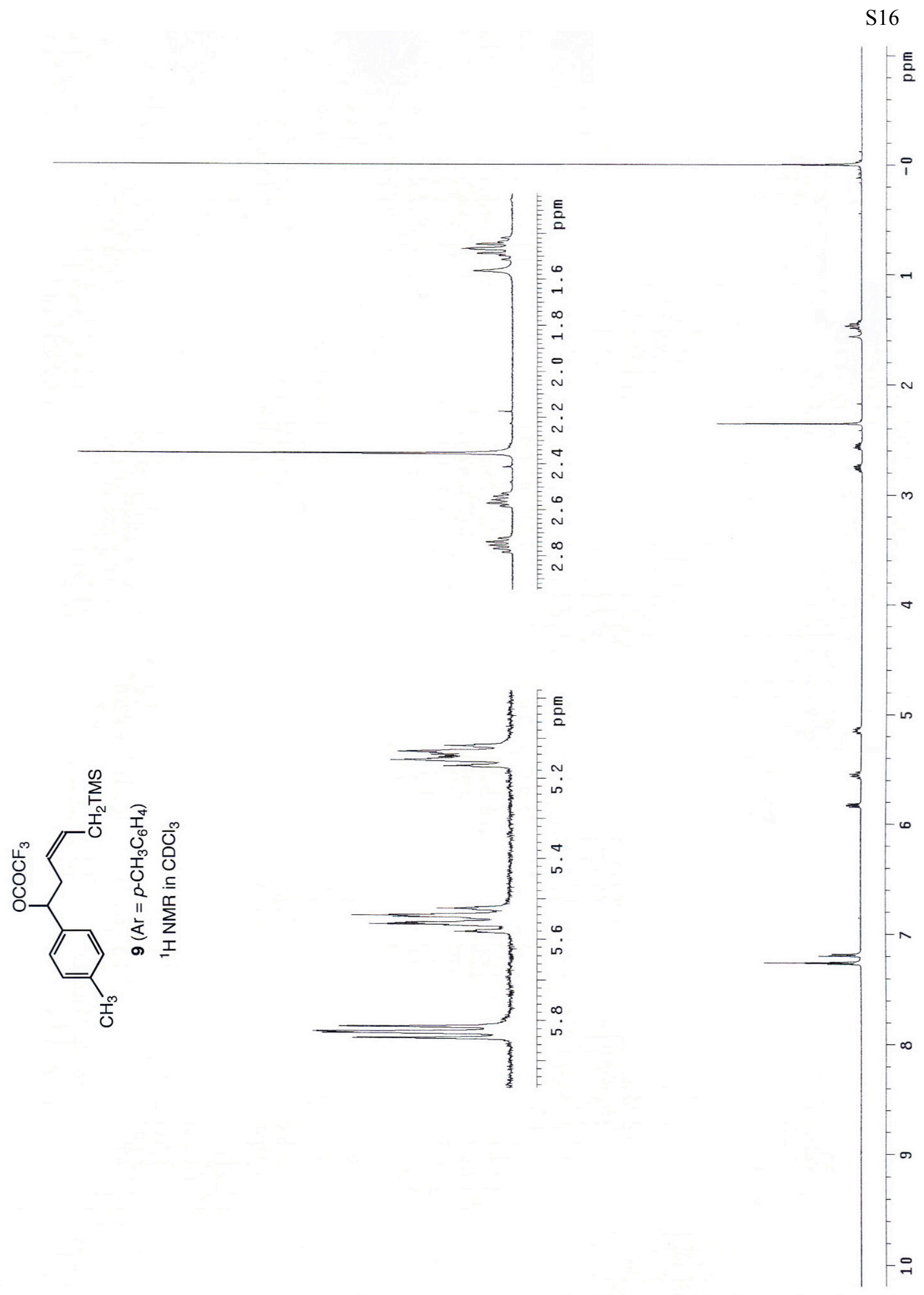




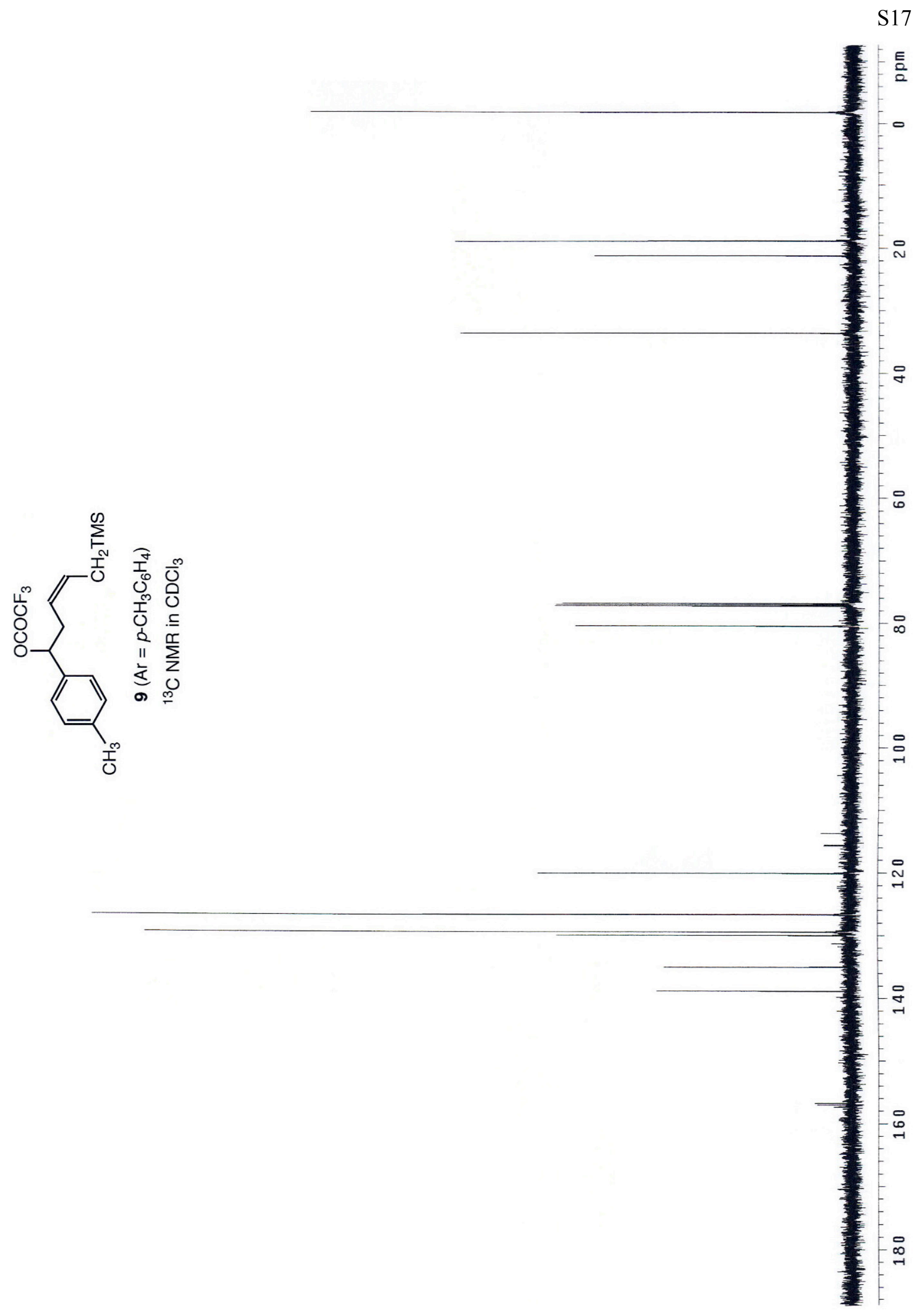




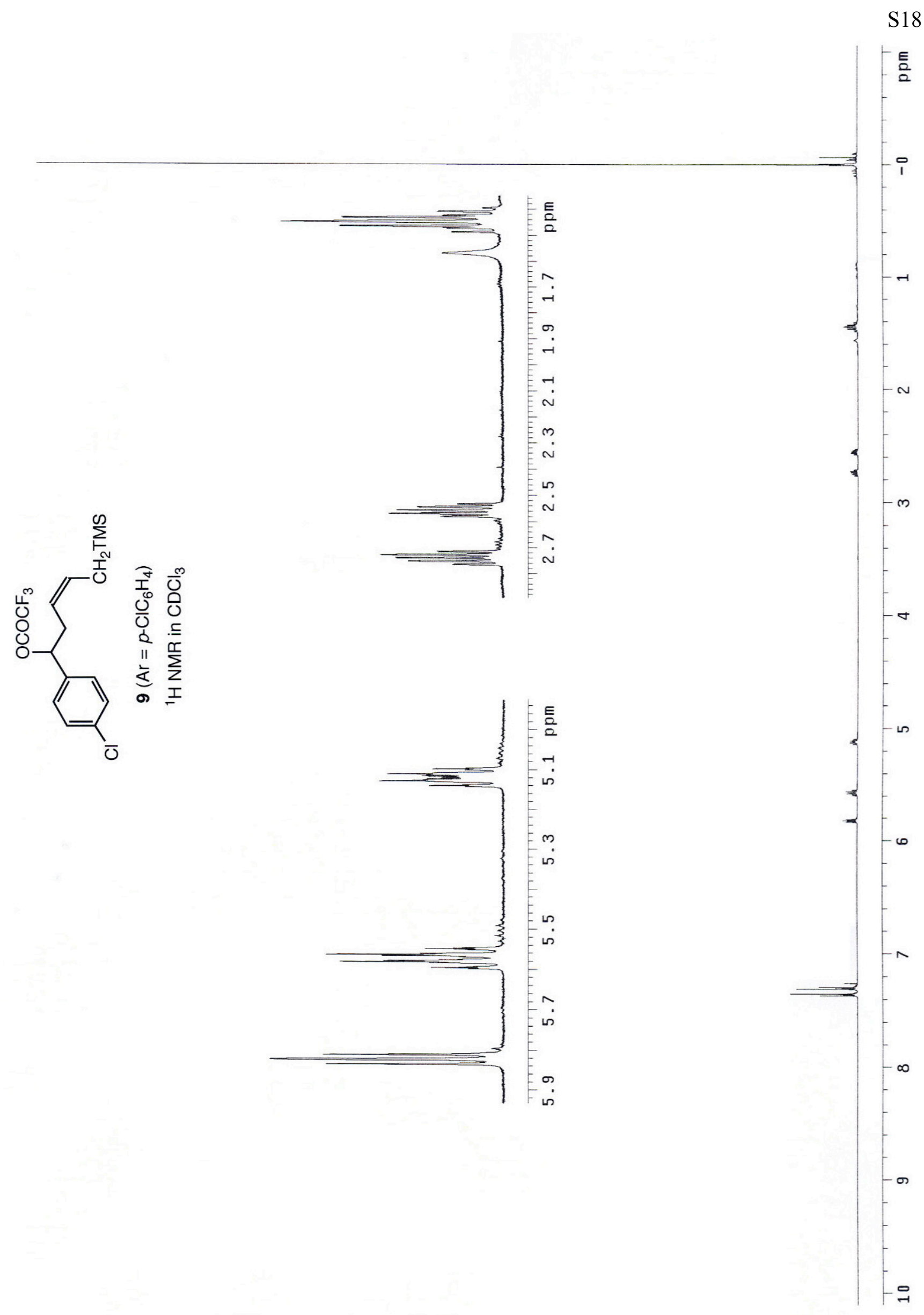




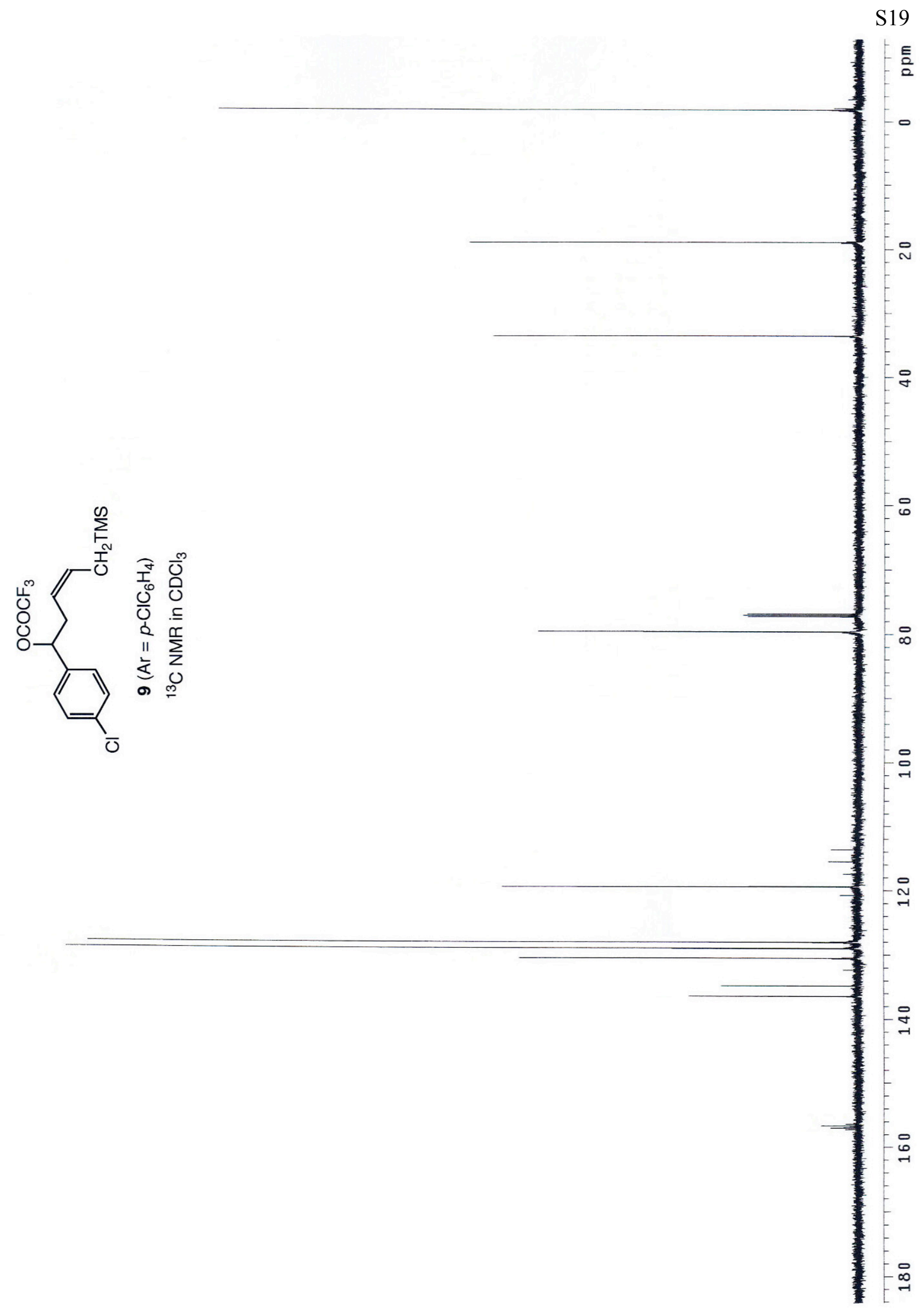




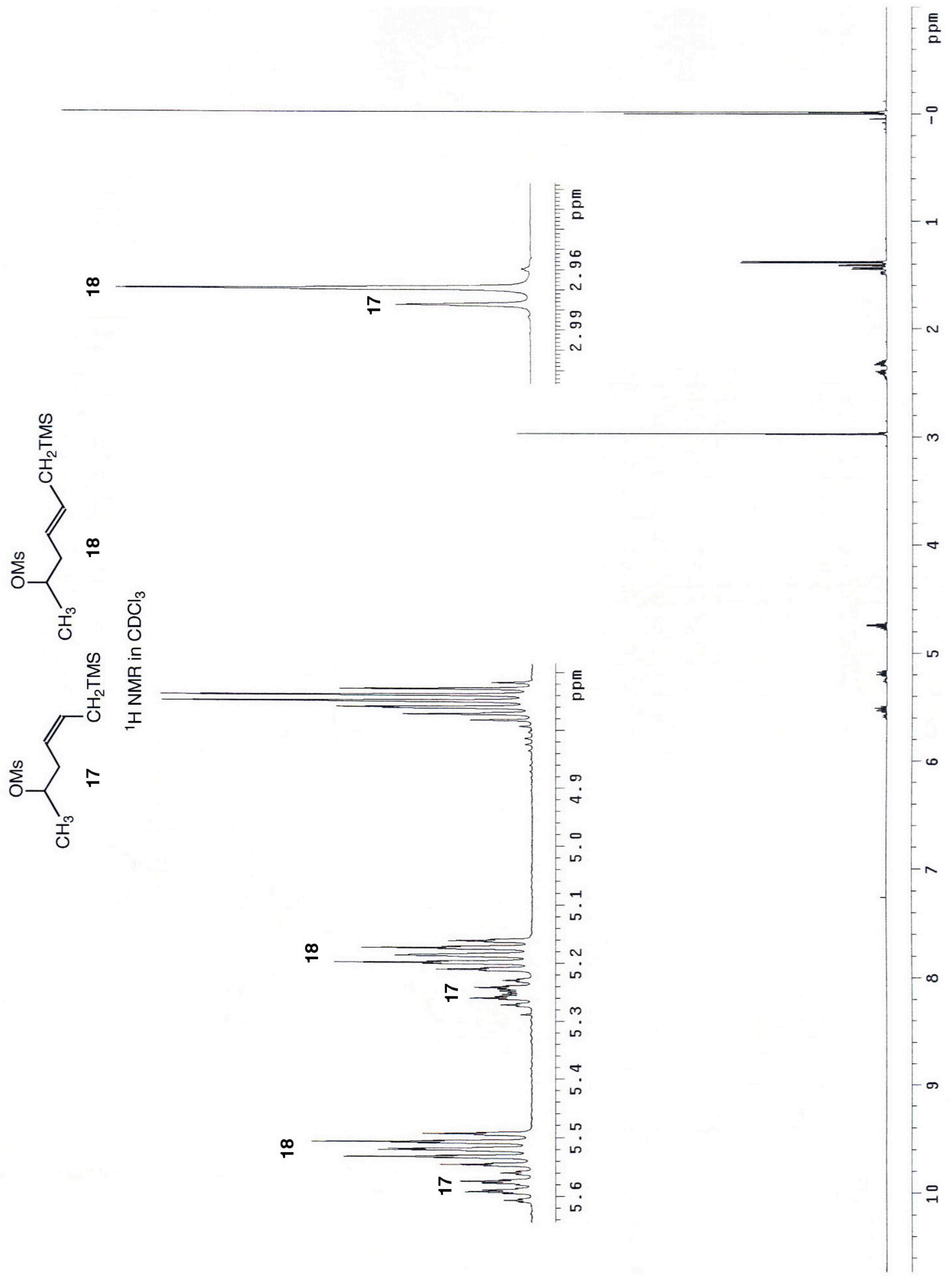




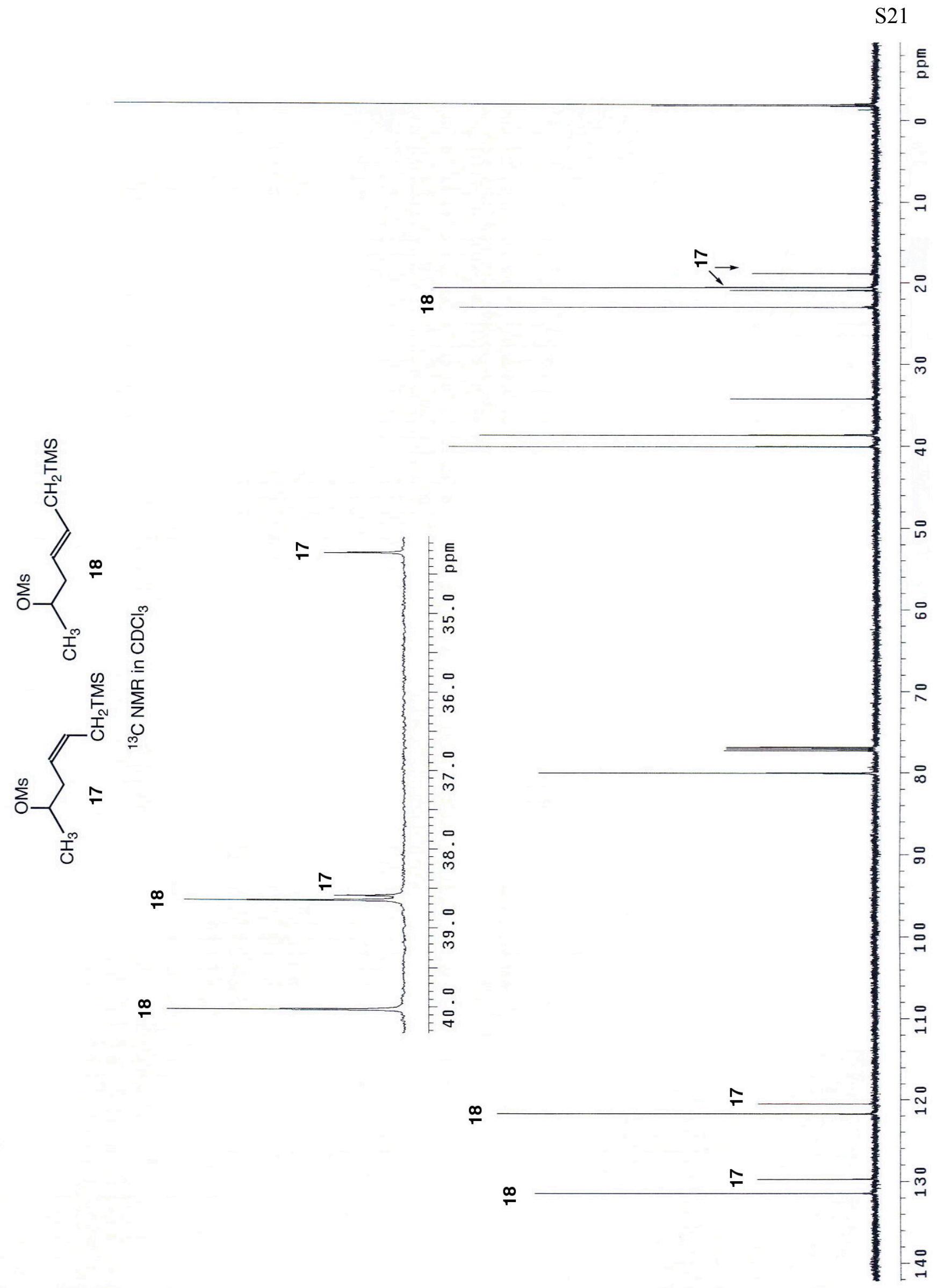




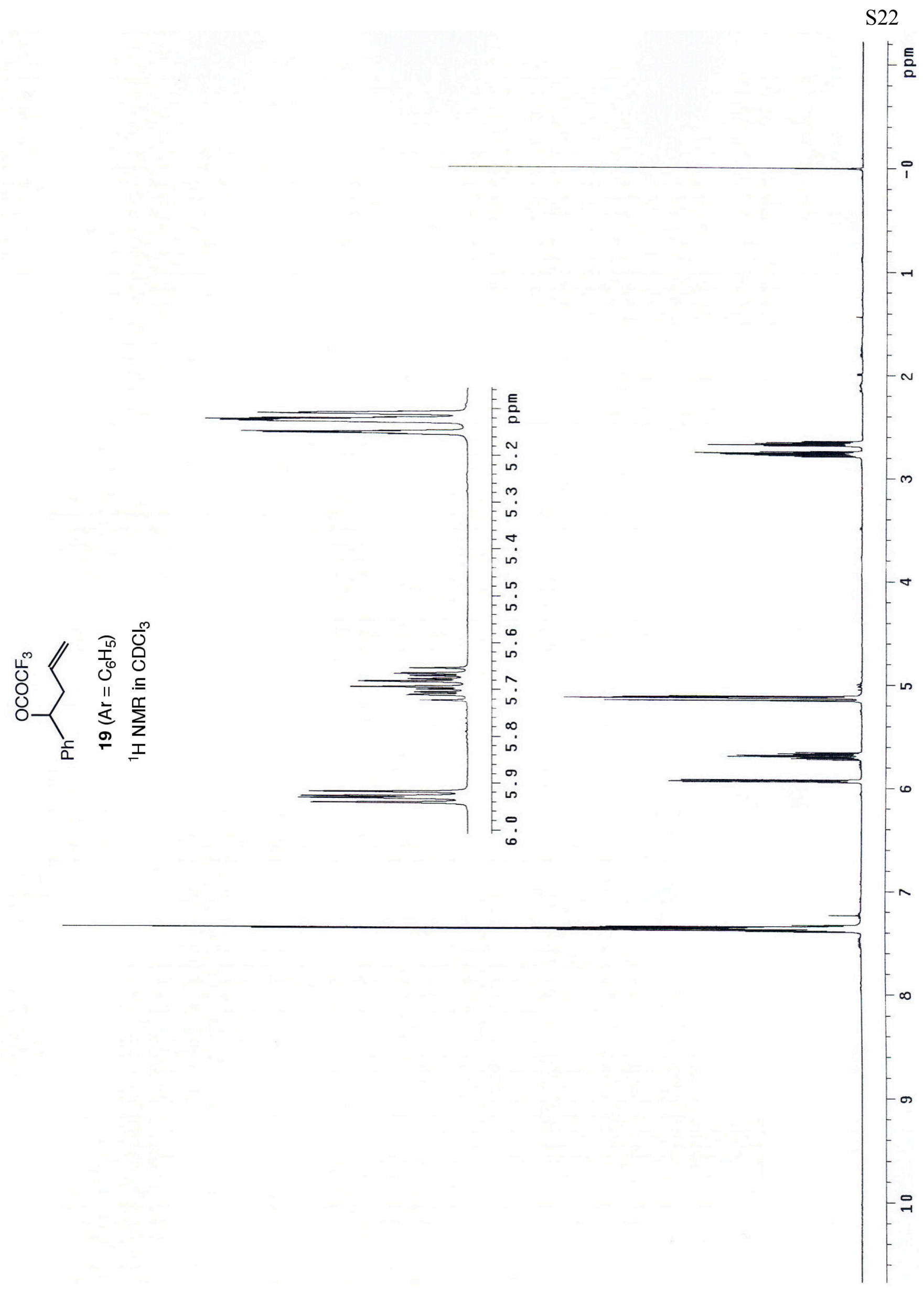




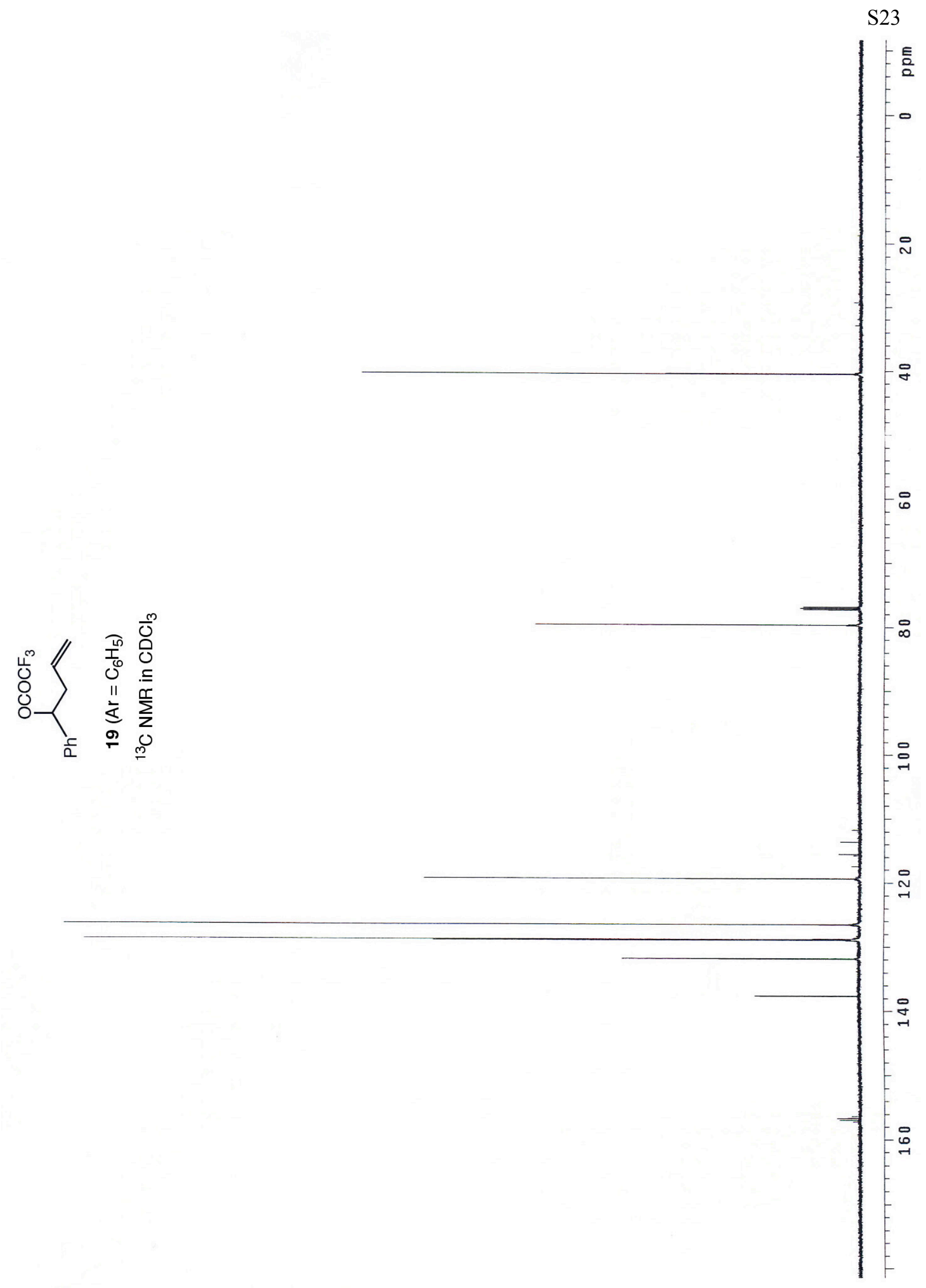




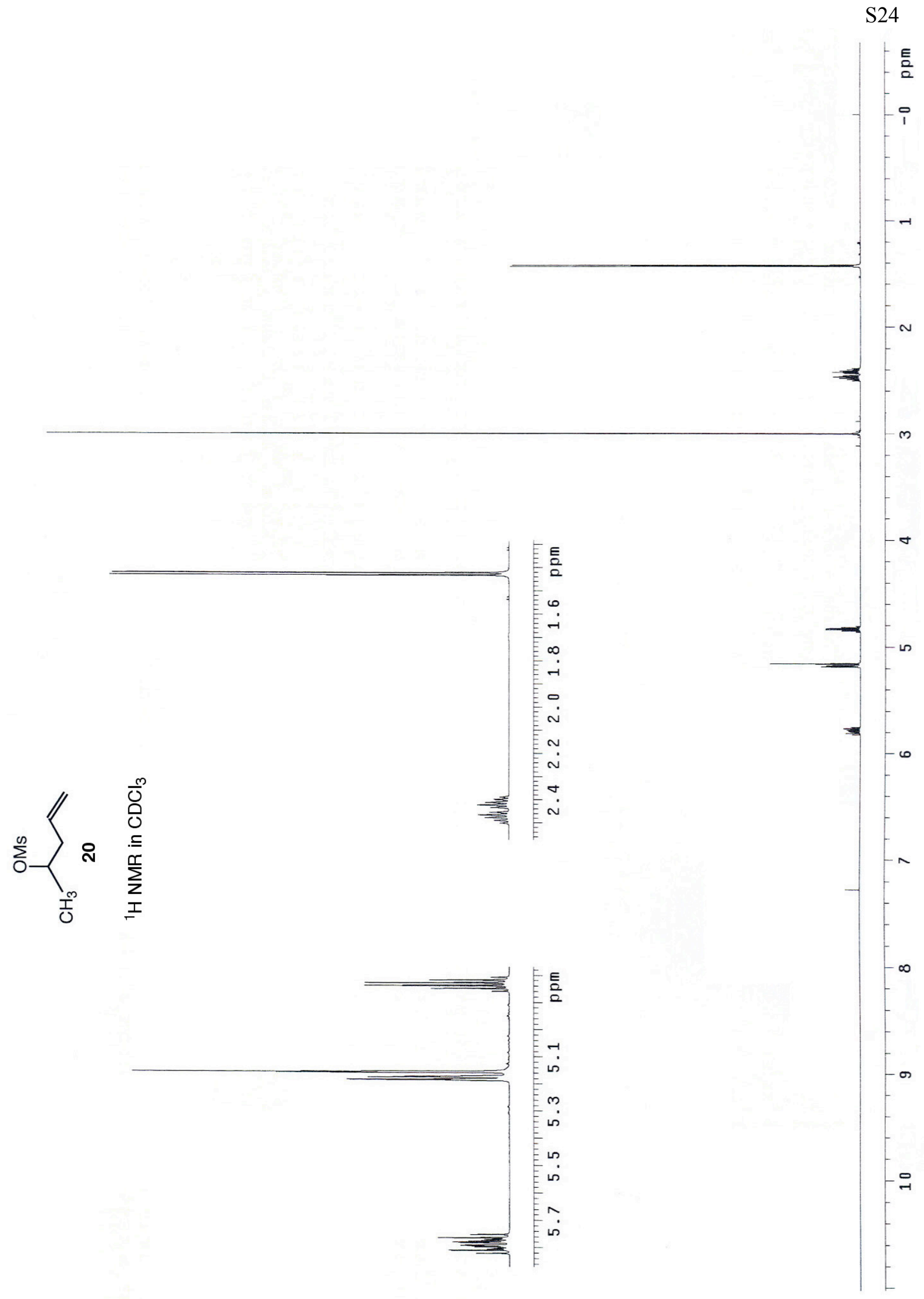




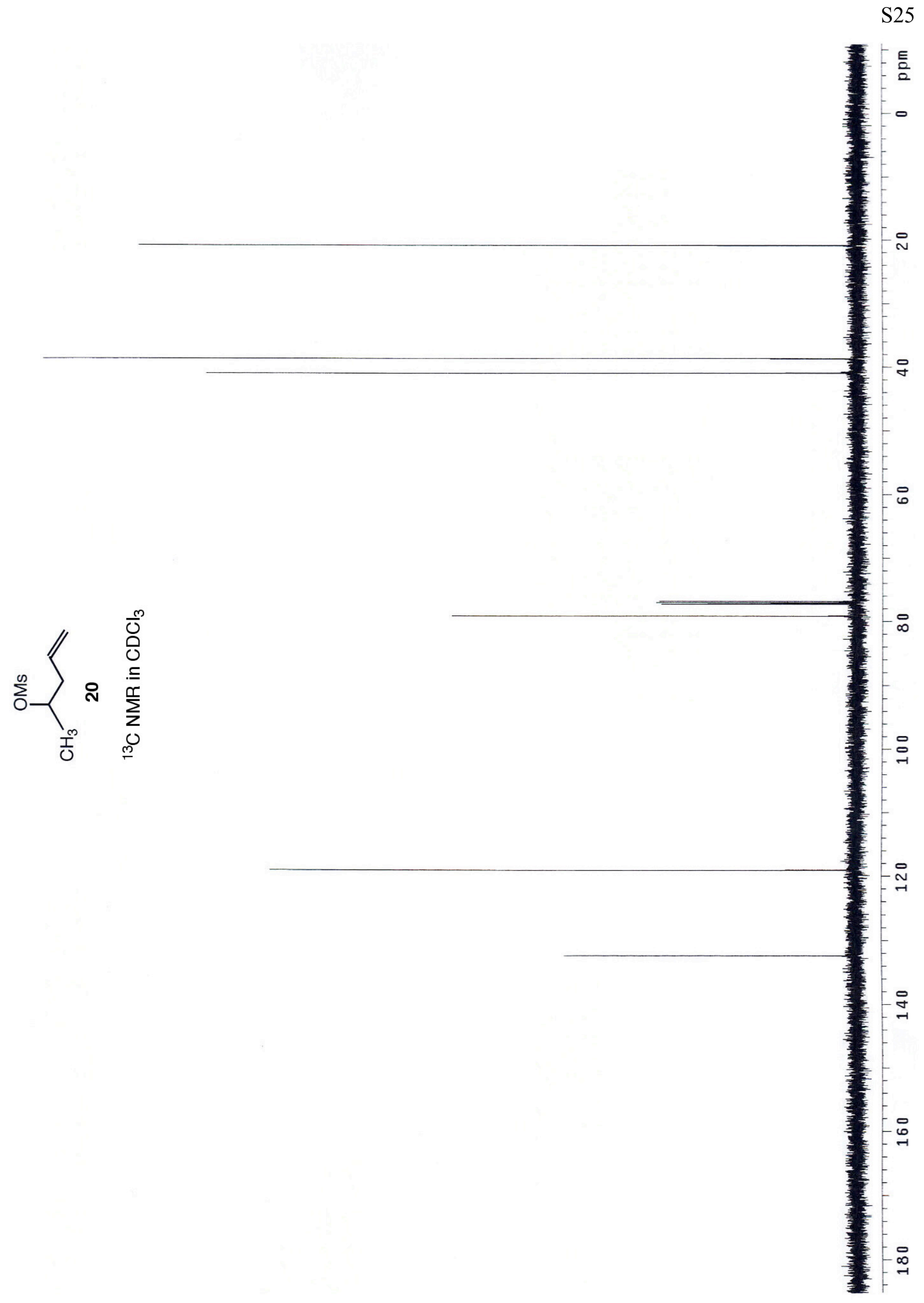




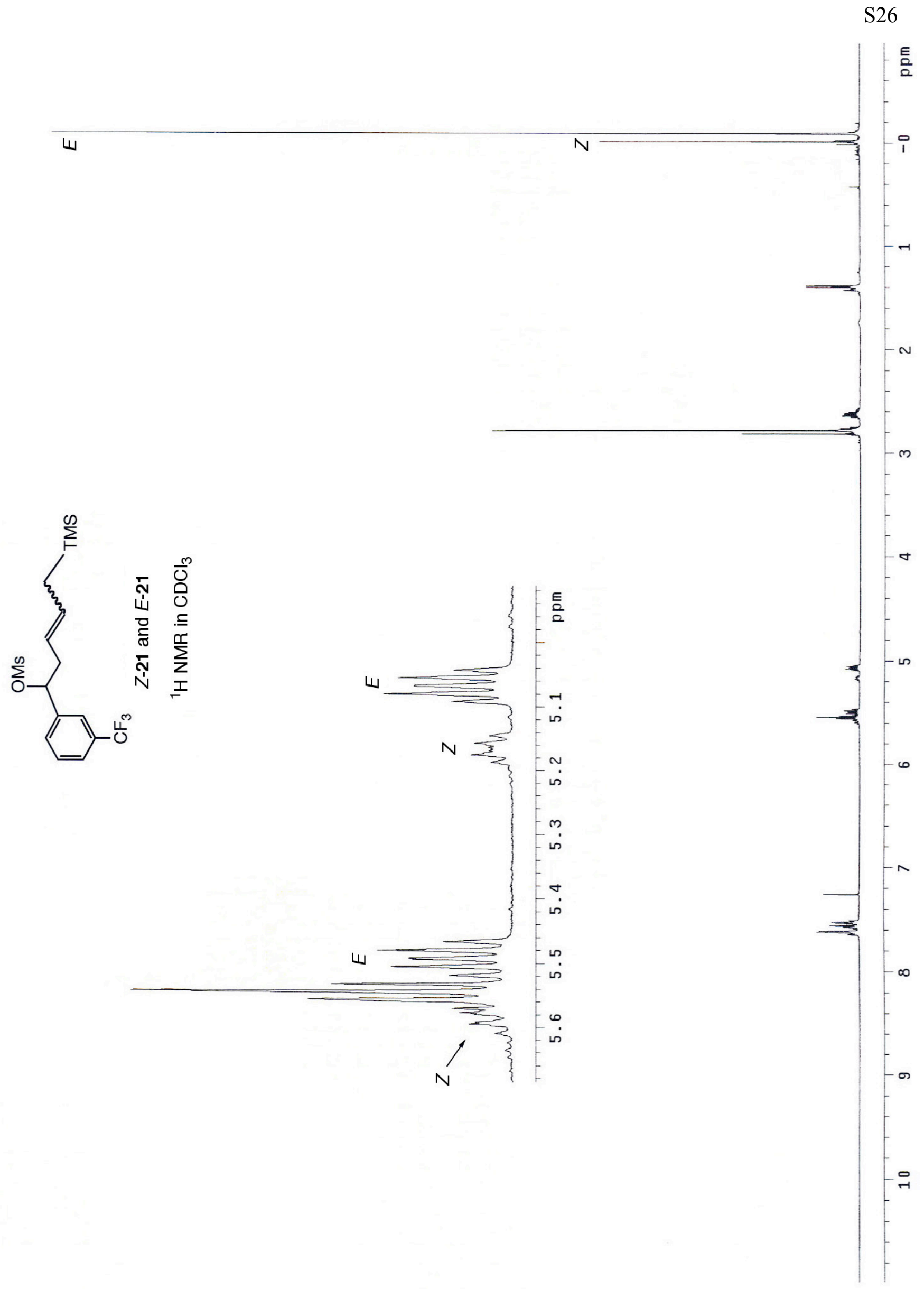




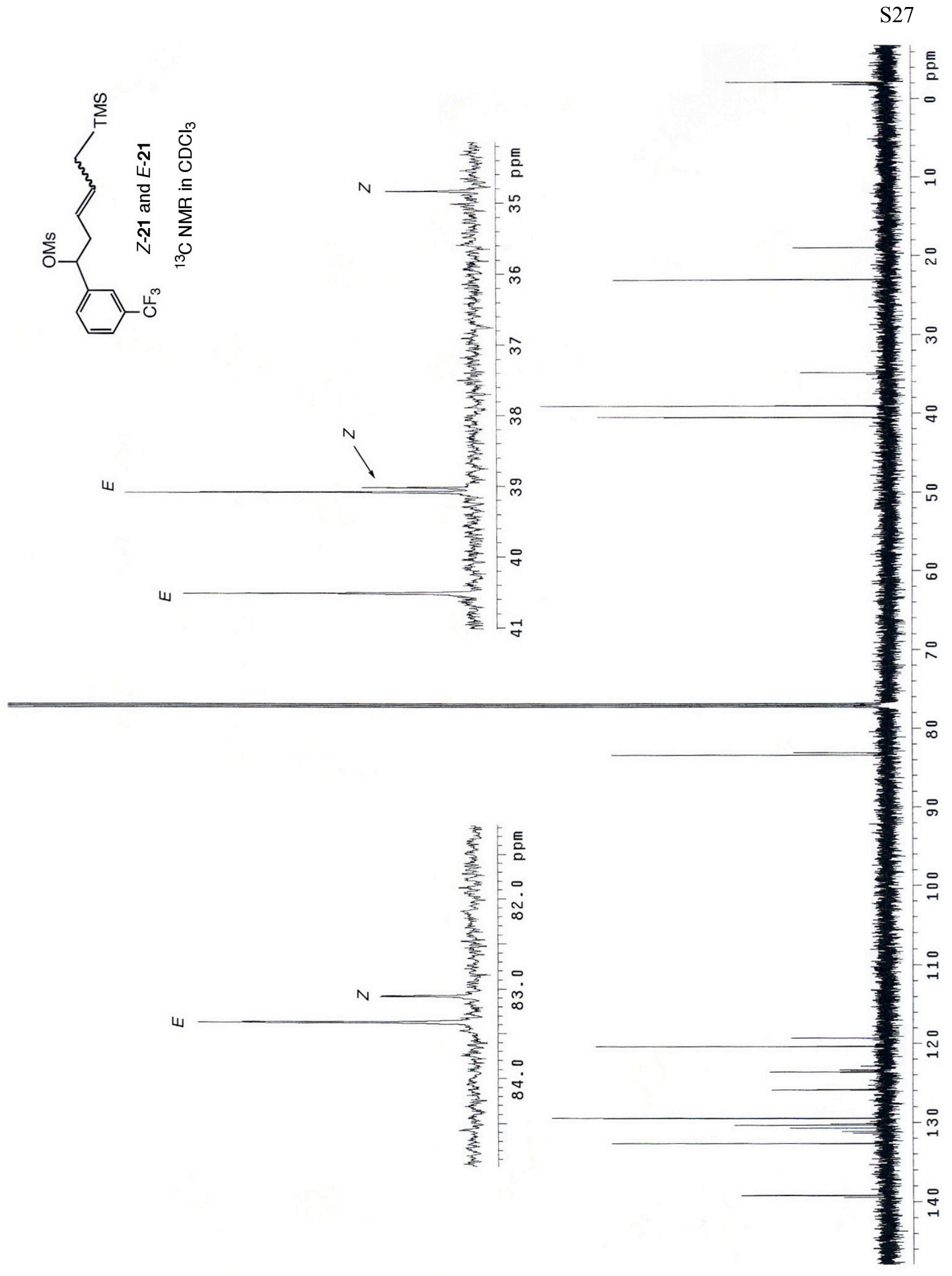




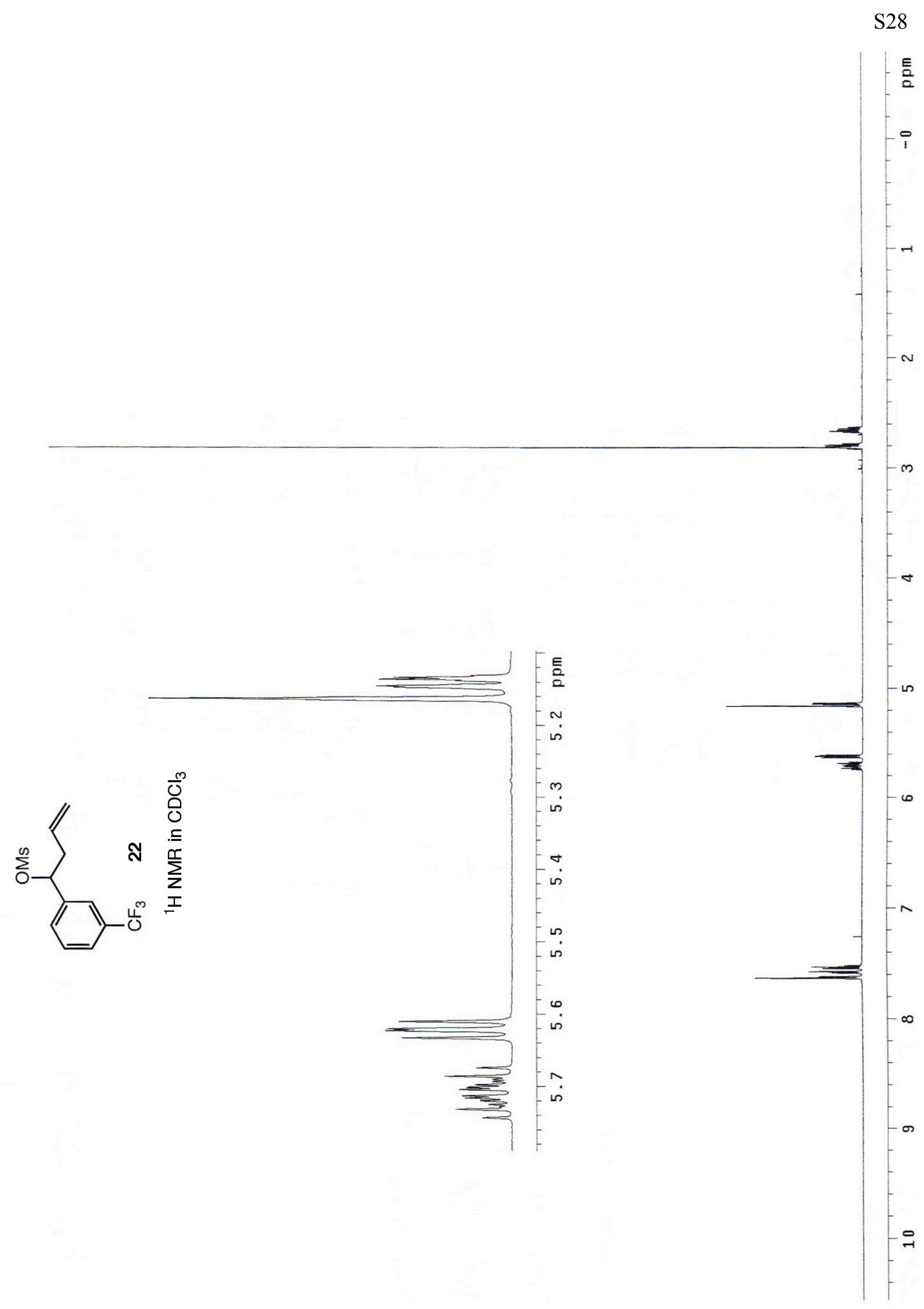




$$
4
$$




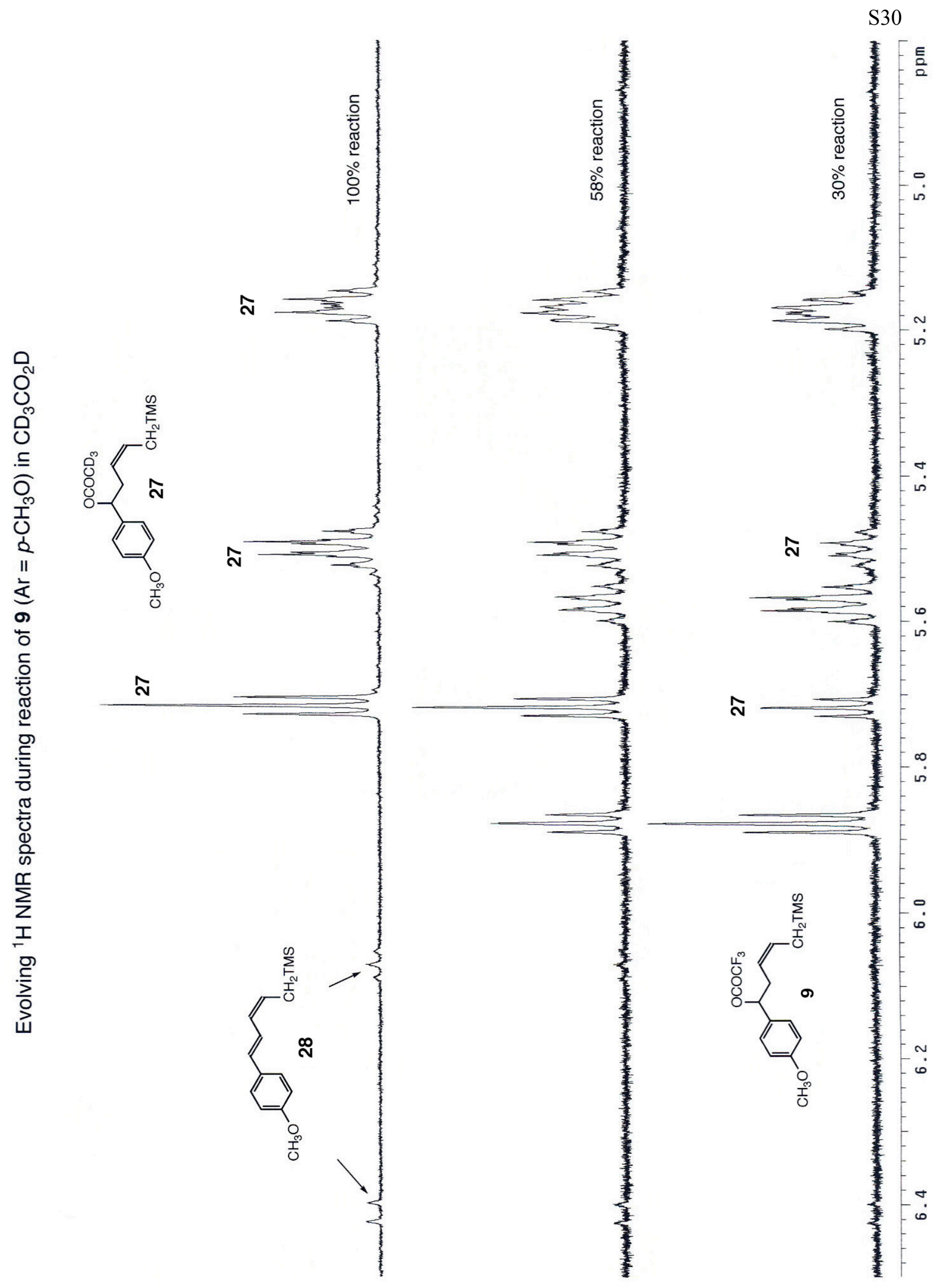




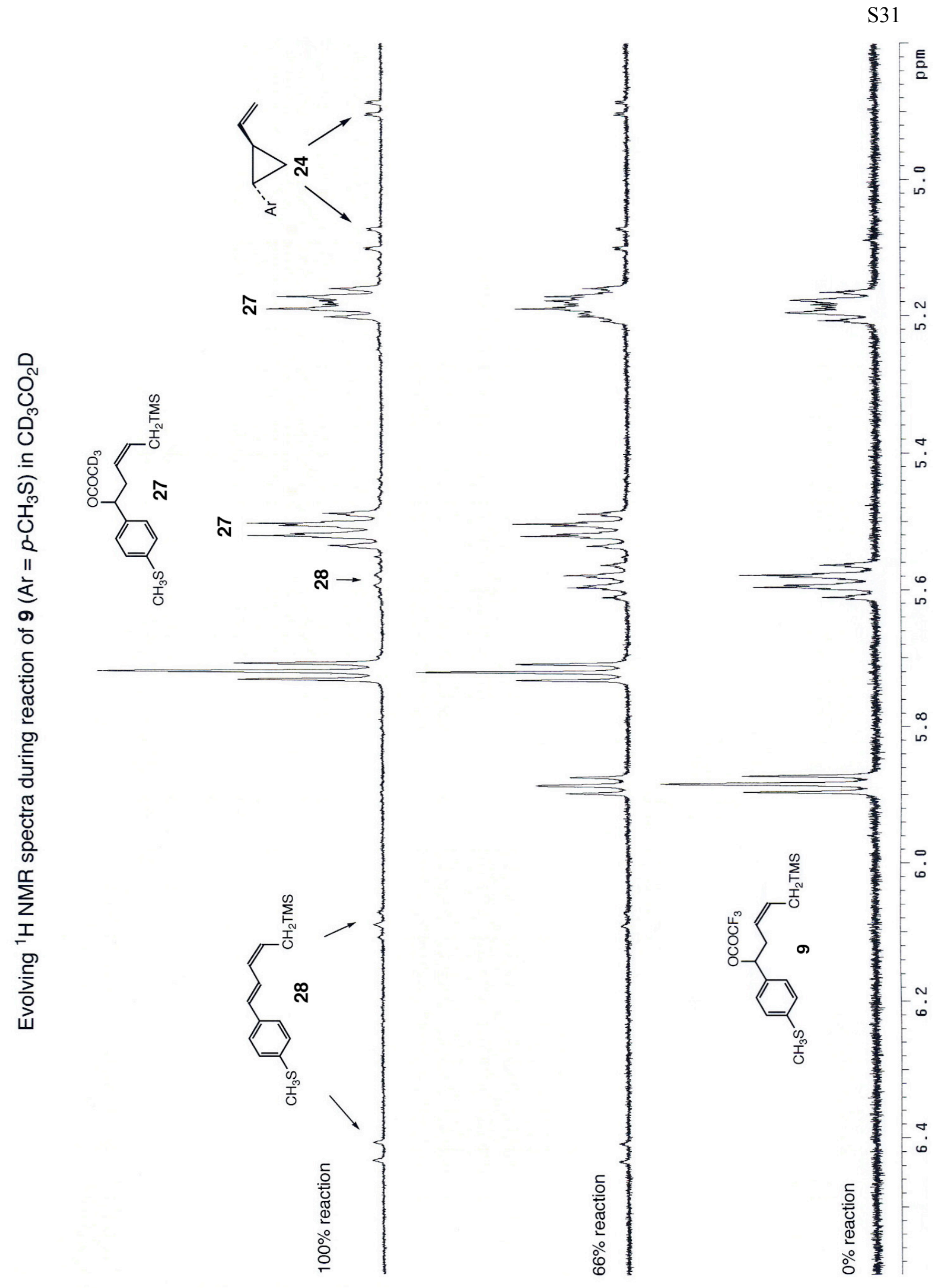




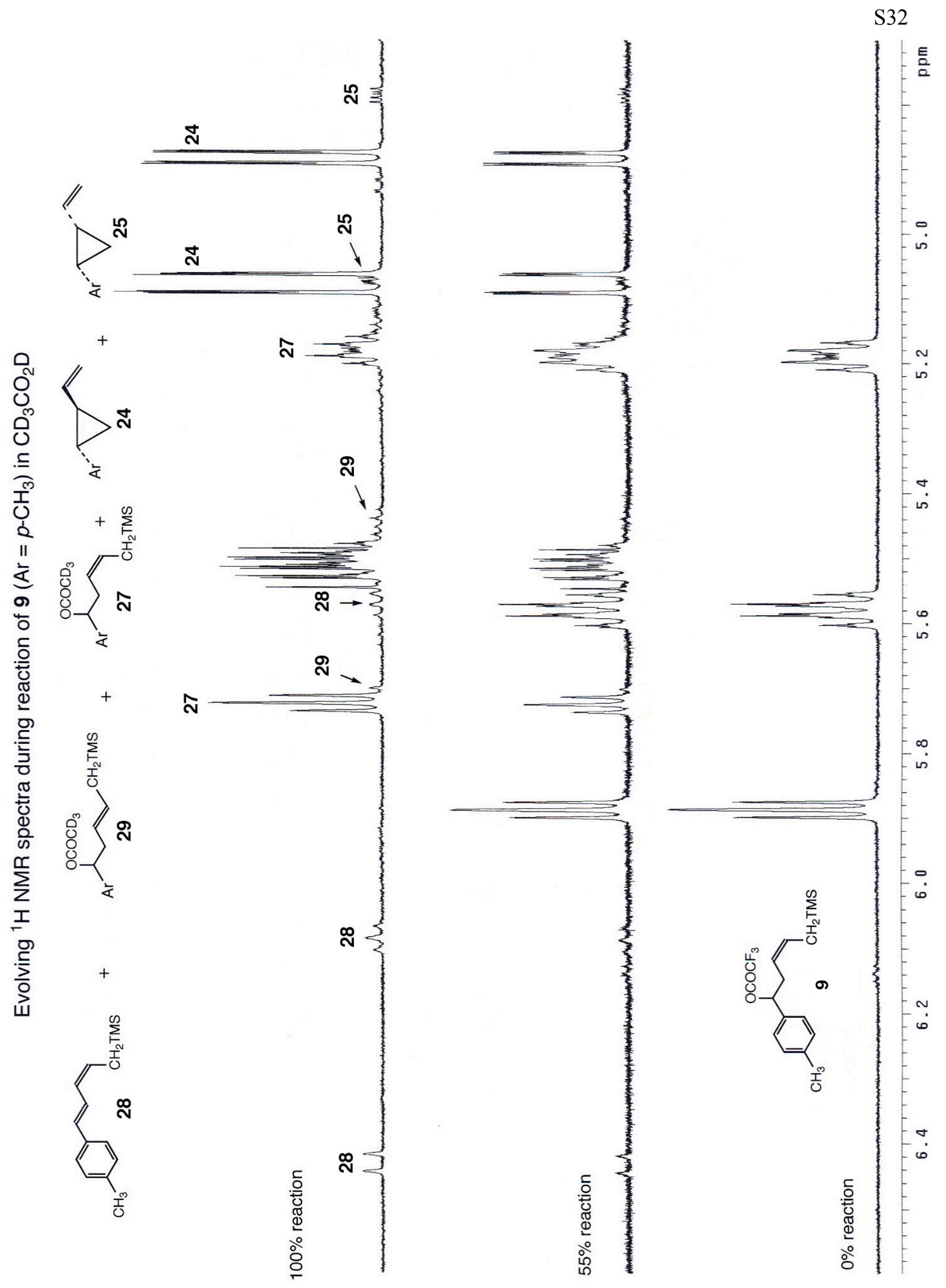




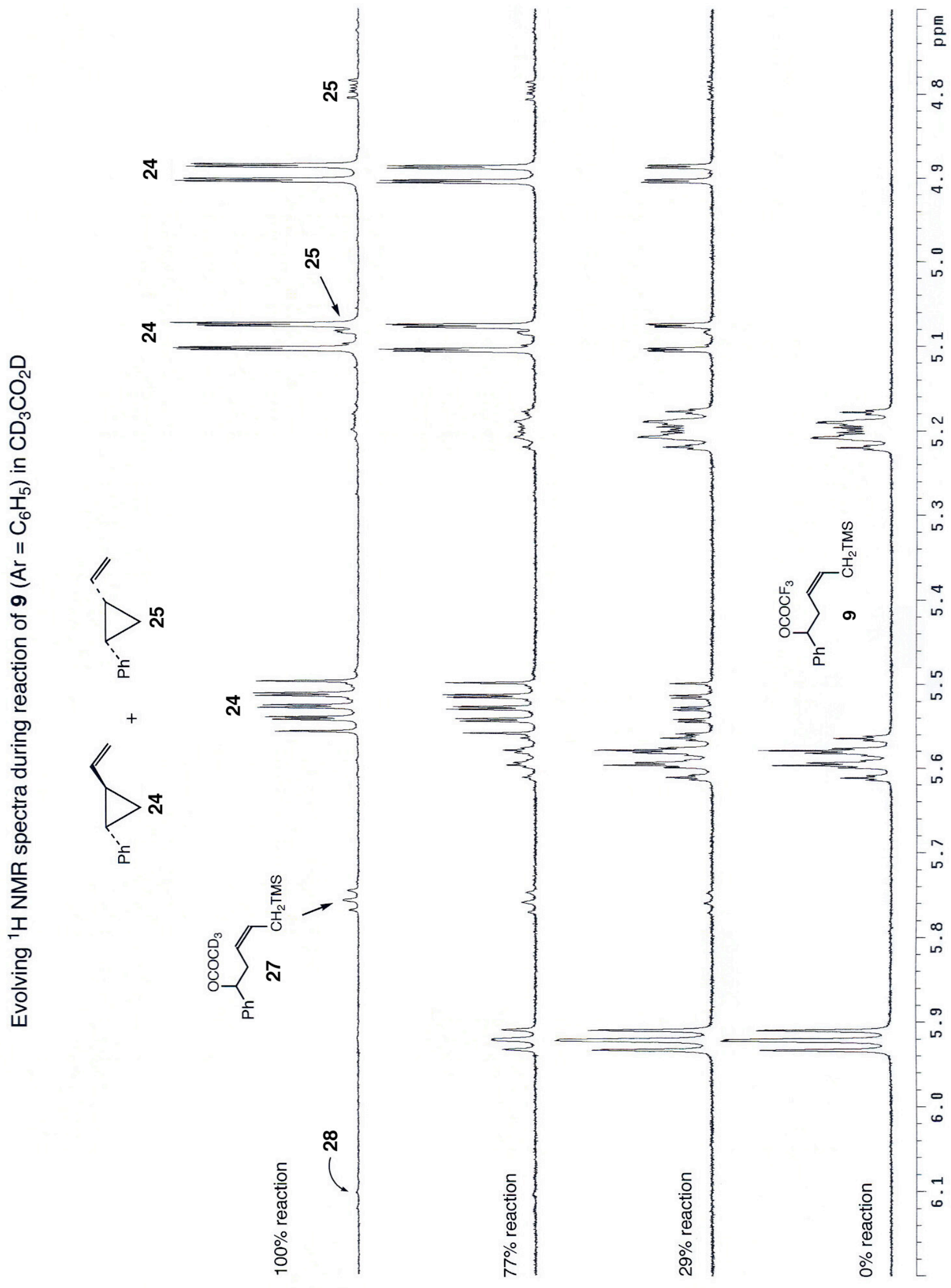




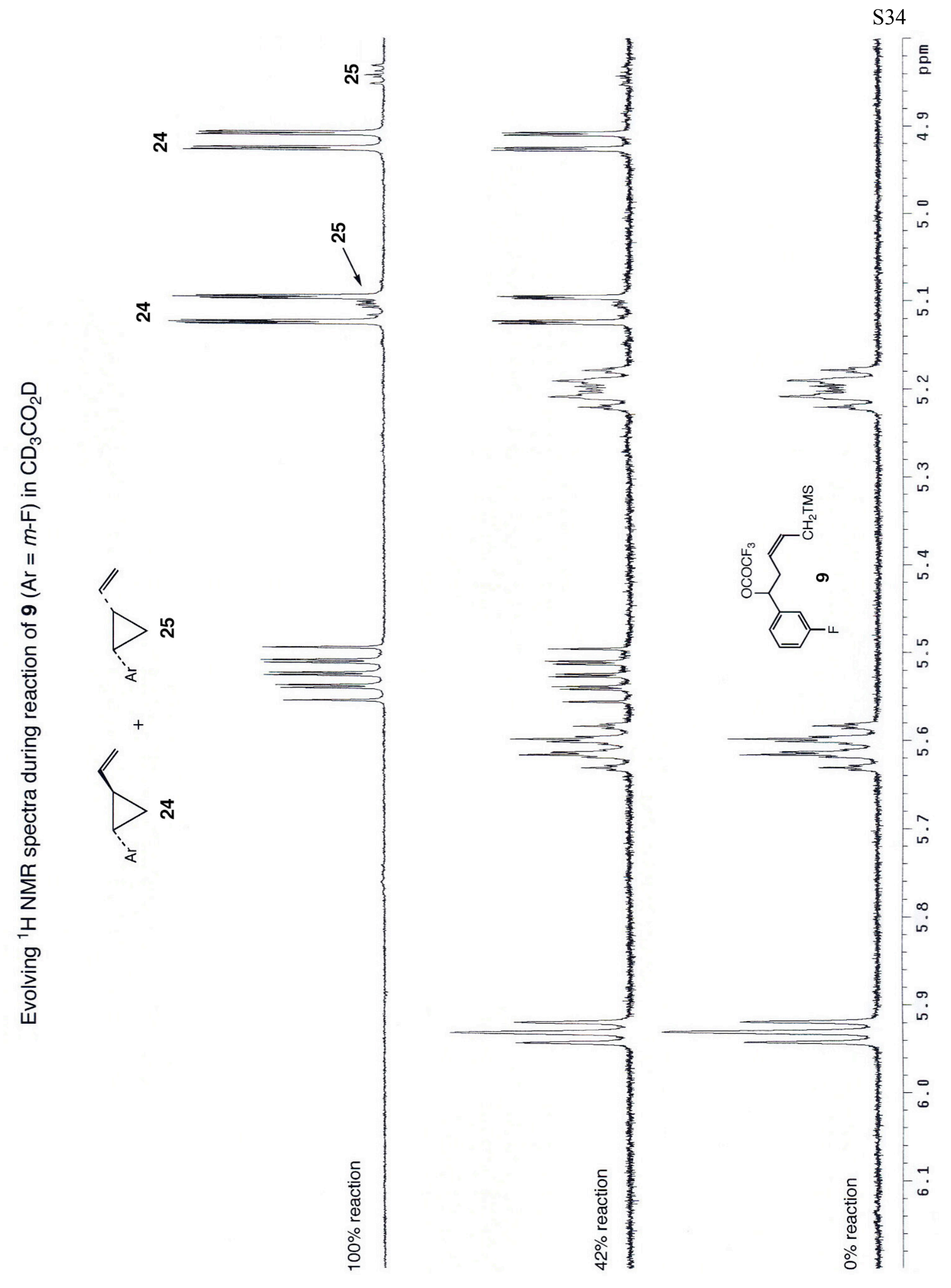




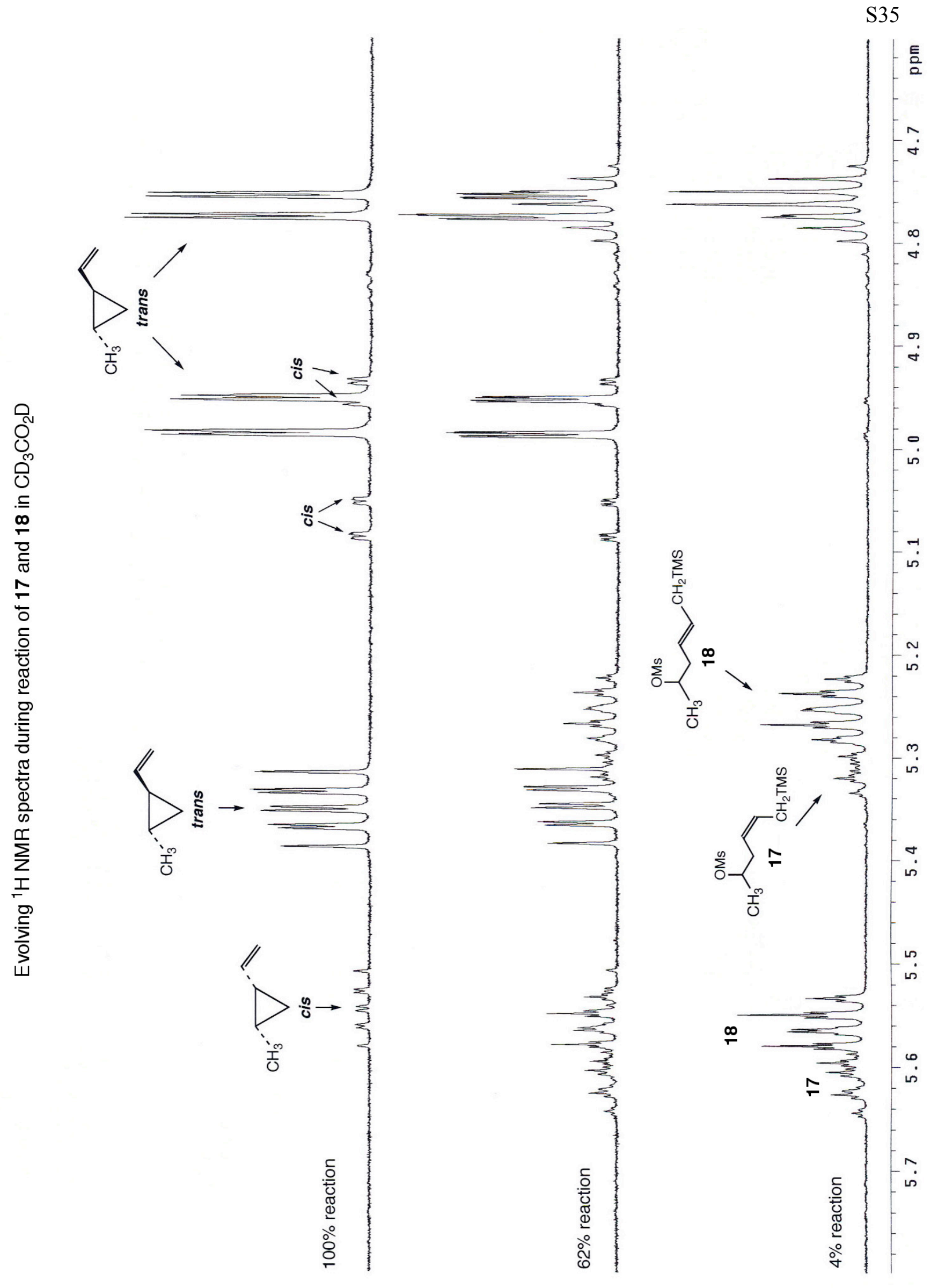



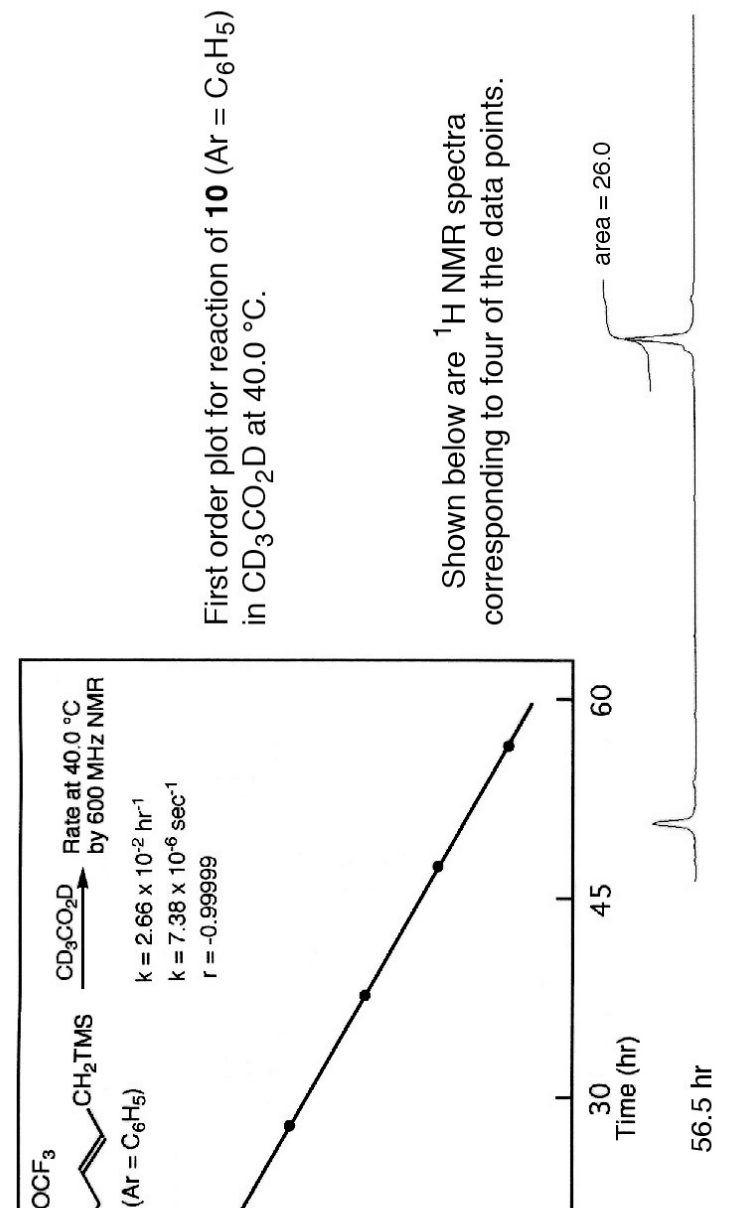
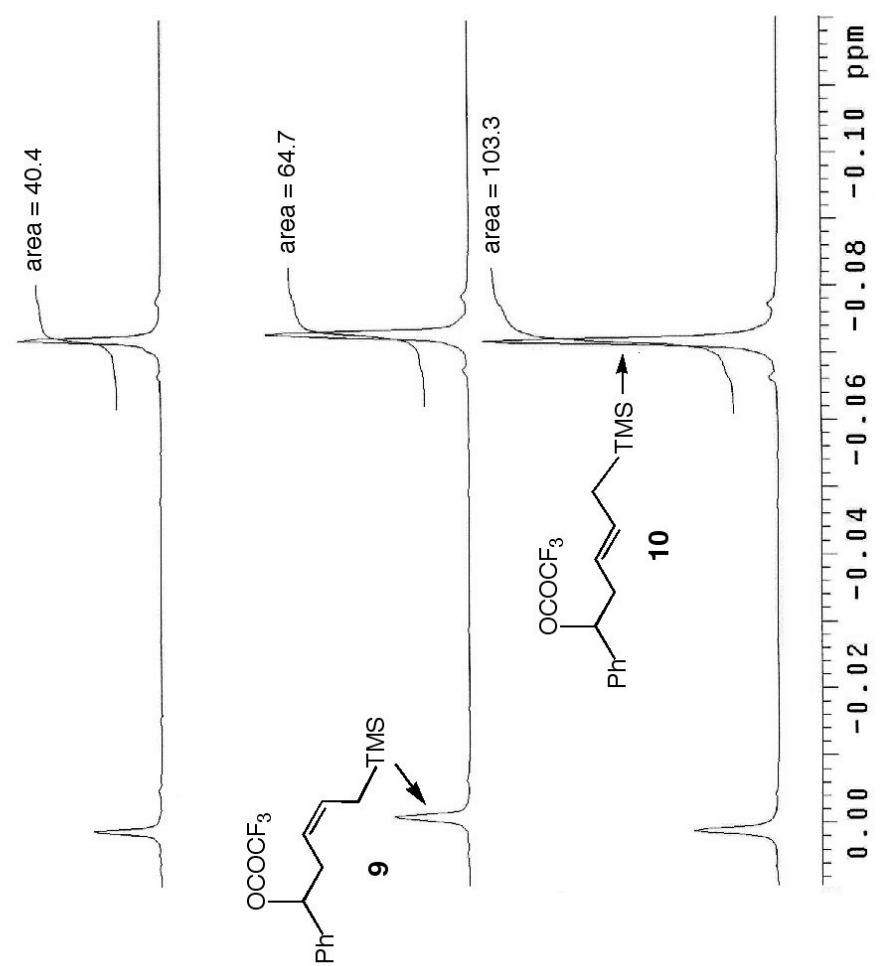

$\stackrel{\overline{1}}{\stackrel{\infty}{\sigma}}$

$\frac{1}{0}$

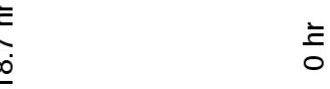

年
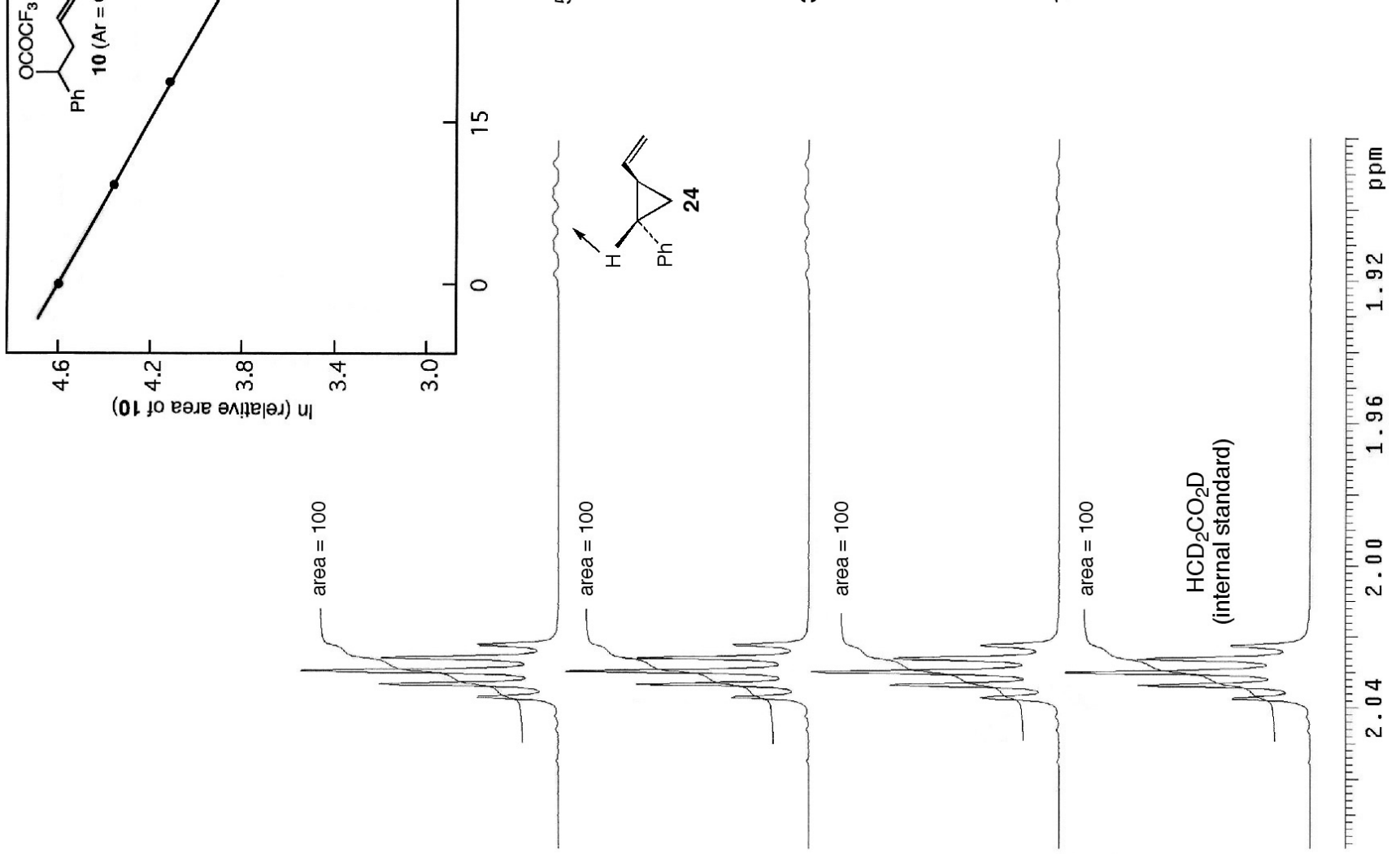